\title{
Critical Assessment of Metagenome Interpretation - the second round of challenges
}

F. Meyer ${ }^{\star 1,2}$, A. Fritz ${ }^{* 1,2,3}$, Z.-L. Deng ${ }^{1,2,4}$, D. Koslicki ${ }^{5}$, A. Gurevich ${ }^{6}$, G. Robertson ${ }^{1,2}$, M. Alser $^{7}$, D. Antipov ${ }^{8}$, F. Beghini ${ }^{9}$, D. Bertrand ${ }^{10}$, J. J. Brito ${ }^{11}$, C.T. Brown ${ }^{12}$, J. Buchmann ${ }^{13}$, A. Buluç ${ }^{14,15}$, B. Chen ${ }^{14,15}$, R. Chikhi ${ }^{16}$, P. T. Clausen ${ }^{17}$, A. Cristian ${ }^{18,19}$, P. W. Dabrowski $^{20,21}$, A. E. Darling ${ }^{22}$, R. Egan ${ }^{23,24}$, E. Eskin ${ }^{25}$, E. Georganas ${ }^{26}$, E. Goltsman ${ }^{23,24}$, M. A. Gray ${ }^{18,27}$, L. H. Hansen ${ }^{28}$, S. Hofmeyr ${ }^{14,15}$, P. Huang ${ }^{29}$, L. Irber ${ }^{12}$, H. Jia ${ }^{30,31}$, T. S. Jørgensen $^{32,33}$, S. D. Kieser ${ }^{34,35}$, T. Klemetsen ${ }^{36}$, A. Kola ${ }^{37}$, M. Kolmogorov ${ }^{38}$, A. Korobeynikov ${ }^{8,39}$, J. Kwan ${ }^{40}$, N. LaPierre ${ }^{25}$, C. Lemaitre ${ }^{41}$, C. Li ${ }^{10}$, A. Limasset ${ }^{42}$, F. Malcher-Miranda $^{43}$, S. Mangul ${ }^{11}$, V. R. Marcelino ${ }^{44,45}$, C. Marchet ${ }^{42}$, P. Marijon ${ }^{46}$, D. Meleshko $^{8}$, D. R. Mende ${ }^{47}$, A. Milanese ${ }^{48,49}$, N. Nagarajan ${ }^{50,51}$, J. Nissen ${ }^{52}$, S. Nurk ${ }^{53}$, L. Oliker $^{14,15}$, L. Paoli48, P. Peterlongo ${ }^{41}$, V. C. Piro ${ }^{43}$, J. S. Porter ${ }^{54}$, S. Rasmussen ${ }^{55}$, E. R. $\operatorname{Rees}^{40}$, K. Reinert ${ }^{56}$, B. Renard ${ }^{57,58}$, E. M. Robertsen ${ }^{36}$, G. L. Rosen ${ }^{18,27,59}$, H.-J. Ruscheweyh $^{48}$, V. Sarwal ${ }^{25}$, N. Segata ${ }^{9}$, E. Seiler ${ }^{56}$, L. Shi ${ }^{60}$, F. Sun ${ }^{61}$, S. Sunagawa ${ }^{48}$, S. J. Sørensen ${ }^{62}$, A. Thomas ${ }^{63,23}$, C. Tong ${ }^{10}$, M. Trajkovski ${ }^{34,64}$, J. Tremblay ${ }^{65}$, G. Uritskiy ${ }^{66}$, R. Vicedomini ${ }^{16}$, Zi. Wang ${ }^{67}$, Zhe. Wang ${ }^{29}$, Zho. Wang ${ }^{68,69,70}$, A. Warren ${ }^{54}$, N. P. Willassen ${ }^{36}$, K. Yelick ${ }^{15,14}$, R. You ${ }^{29}$, G. Zeller ${ }^{49}$, Z. Zhao ${ }^{18}$, S. Zhu ${ }^{71,72}$, J. Zhu ${ }^{30,31}$, R. Garrido-Oter $^{73}$, P. Gastmeier ${ }^{37}$, S. Hacquard ${ }^{73}$, S. Häußler ${ }^{74}$, A. Khaledi ${ }^{74}$, F. Maechler ${ }^{37}$, F. Mesny ${ }^{73}$, S. Radutoiu ${ }^{75}$, P. Schulze-Lefert ${ }^{73}$, N. Smit ${ }^{74}$, T. Strowig ${ }^{74}$, A. Bremges ${ }^{1,3}$, A. Sczyrba ${ }^{76}$, A. C. McHardy $\# 1,2,3,4,77$

${ }^{1}$ Computational Biology of Infection Research, Helmholtz Centre for Infection Research, Braunschweig, Germany. 'Braunschweig Integrated Centre of Systems Biology (BRICS), Braunschweig, Germany. ${ }^{3}$ German Center for Infection Research (DZIF), HannoverBraunschweig site. ${ }^{4}$ Cluster of Excellence RESIST (EXC 2155), Hannover Medical School, Hannover, Germany. ${ }^{5}$ Pennsylvania State University, State College, United States. ${ }^{6}$ St. Petersburg State University, St. Petersburg, Russia. ${ }^{7}$ Department of Information Technology and Electrical Engineering, ETH Zürich, Zurich, Switzerland. ${ }^{8}$ Center for Algorithmic Biotechnology, Saint Petersburg State University, Saint Petersburg, Russia. ${ }^{9}$ Department CIBIO, University of Trento, Trento, Italy. ${ }^{10} \mathrm{Genome}$ Institute of Singapore, Singapore, Singapore. ${ }^{11}$ University of Southern California, Los Angeles, United States. ${ }^{12}$ University of California, Davis, Davis, United States. ${ }^{13}$ Institute for Biological Data Science, Heinrich-Heine-University, Düsseldorf, Germany. ${ }^{14}$ Lawrence Berkeley National Laboratory, Berkeley, United States. ${ }^{15}$ University of California, Berkeley, Berkeley, United States. ${ }^{16}$ Institut Pasteur, Paris, France. ${ }^{17}$ National Food Institute, Division of global surveillance, Technical University of Denmark, Lyngby, Denmark. ${ }^{18}$ Drexel University, Philadelphia, United States. ${ }^{19} \mathrm{Google}$ Inc., Philadelphia, United States. ${ }^{20}$ Robert KochInstitut, Berlin, Germany. ${ }^{21}$ Hochschule für Technik und Wirtschaft Berlin, Berlin, Germany. 
${ }^{22}$ University of Technology Sydney, Sydney, Australia. ${ }^{23} \mathrm{DOE}$ Joint Genome Institute, Berkeley, United States. ${ }^{24}$ Lawrence Berkeley National Labs, Berkeley, United States. ${ }^{25}$ University of California, Los Angeles, Los Angeles, United States. ${ }^{26}$ Intel Corporation, Santa Clara, United States. ${ }^{27}$ Ecological and Evolutionary Signal-processing and Informatics (EESI) lab, Philadelphia, United States. ${ }^{28}$ University of Copenhagen, Department of Plant and Environmental Science, Thorvaldsensvej 40, 1871 Frederiksberg, Denmark. ${ }^{29}$ School of Computer Science, Fudan University, Shanghai, China. ${ }^{30} \mathrm{BGI}-S h e n z h e n$, Shenzhen, China. ${ }^{31}$ Shenzhen Key Laboratory of Human Commensal Microorganisms and Health Research, BGI-Shenzhen, Shenzhen, China. ${ }^{32}$ Technical University of Denmark, Novo Nordisk Foundation Center for Biosustainability, Kemitorvet, 220, 328D, 2800 Kgs. Lyngby, Denmark. ${ }^{33}$ Aarhus University, Department of Environmental Science, Frederiksborgvej 399, 4000 Roskilde, Denmark. ${ }^{34}$ Department of Cell Physiology and Metabolism, Faculty of Medicine, University of Geneva, Geneva, Switzerland. ${ }^{35}$ Swiss Institute of Bioinformatics, Geneva, Switzerland. ${ }^{36}$ The Arctic University of Norway, Tromsø, Norway. ${ }^{37}$ Charité - Universitätsmedizin Berlin, Berlin, Germany. ${ }^{38}$ Department of Comuter Science and Engineering, University of California San Diego, San Diego, United States. ${ }^{39}$ Department of Statistical Modelling, Saint Petersburg State University, Saint Petersburg, Russia. ${ }^{40}$ University of Wisconsin - Madison, Madison, United States. ${ }^{41}$ Univ. Rennes, Inria, CNRS, IRISA, Rennes, France. ${ }^{42}$ Université Lille, CNRS, CRIStAL , Lille, France. ${ }^{43}$ Hasso Plattner Institute, Digital Engineering Faculty, University of Potsdam, Potsdam, Germany. ${ }^{44}$ Sydney Medical School, The University of Sydney, Sydney, Australia. ${ }^{45}$ Centre for Innate Immunity and Infectious Diseases, Hudson Institute of Medical Research, Clayton, Australia. ${ }^{46}$ Department of Computer Science, Inria, Univ. Lille, CNRS, Centrale Lille, UMR 9189 - CRIStAL, Lille, France. ${ }^{47}$ Amsterdam University Medical Center, Amsterdam, The Netherlands. ${ }^{48}$ Department of Biology, Institute of Microbiology and Swiss Institute of Bioinformatics, ETH Zürich, Zürich, Switzerland. ${ }^{49}$ Structural and Computational Biology Unit, EMBL, Zürich, Switzerland. ${ }^{50}$ Genome Institute of Singapore, A*STAR, Singapore, Singapore. ${ }^{51}$ National University of Singapore, Singapore, Singapore. ${ }^{52}$ DTU Health Tech, Kongens Lyngby, Denmark. ${ }^{53}$ Genome Informatics Section, Computational and Statistical Genomics Branch, National Human Genome Research Institute, National Institutes of Health, Bethesda, MD, United States, Bethesda, United States. ${ }^{54}$ University of Virginia, Charlottesville, United States. ${ }^{55}$ Novo Nordisk Foundation Center for Protein Research, Faculty of Health and Medical Sciences, University of Copenhagen, Copenhagen, Denmark. ${ }^{56}$ Institute for Bioinformatics, FU Berlin, Berlin, Germany. ${ }^{57}$ Hasso Plattner Institute, Digital Engineering Faculty, University of Potsdam, Potsdam, Germany, Potsdam, Germany. ${ }^{58}$ Bioinformatics Unit (MF1), Robert Koch Institute, Berlin, Germany. ${ }^{59}$ Center for Biological Discovery from Big Data, Philadelphia, United States. ${ }^{60}$ Florida Polytechnic University, Lakeland, United States. ${ }^{61}$ Quantitative and Computational Biology Department, University of Southern California, Los Angeles, United States. ${ }^{62}$ University of Copenhagen, Copenhagen, Denmark. ${ }^{63}$ University of British Columbia, Vancouver, Canada. ${ }^{64}$ Diabetes Center, Faculty of Medicine, University of Geneva, Geneva, Switzerland. ${ }^{65}$ National Research Council Canada, Montreal, Canada. ${ }^{66}$ Phase Genomics, Seattle, United States. ${ }^{67}$ School of Mathematical Sciences, Fudan University, Shanghai, China. ${ }^{68}$ Department of Energy Joint Genome Institute, Berkeley, CA, United States. ${ }^{69}$ Environmental Genomics and SystemsBiology Division, Lawrence Berkeley National Laboratory, Berkeley, CA, United States. ${ }^{70}$ School of Natural Sciences, University of California at Merced, Merced, CA, United States, Berkeley, USA. ${ }^{71}$ Institute of Science and 
Technology for Brain-Inspired Intelligence, Fudan University, Shanghai, China. ${ }^{72}$ Key Laboratory of Computational Neuroscience and Brain-Inspired Intelligence (Fudan University), Ministry of Education, Shanghai, China. ${ }^{73}$ Max Planck Institute for Plant Breeding Research, Köln, Germany. ${ }^{74}$ Helmholtz Centre for Infection Research, Braunschweig, Germany. ${ }^{75}$ Aarhus University, Aarhus, Denmark. ${ }^{76}$ Center for Biotechnology (CeBiTec), Bielefeld University, Bielefeld, Germany. ${ }^{77}$ Technische Universität Braunschweig, Braunschweig, Germany.

*Shared first authors

\#Corresponding author: A. C. McHardy, Department Computational Biology of Infection Research, Helmholtz Centre for Infection Research, Braunschweig, Germany

Email: amc14@helmholtz-hzi.de 


\section{Abstract}

Evaluating metagenomic software is key for optimizing metagenome interpretation and focus of the community-driven initiative for the Critical Assessment of Metagenome Interpretation (CAMI). In its second challenge, CAMI engaged the community to assess their methods on realistic and complex metagenomic datasets with long and short reads, created from $\sim 1,700$ novel and known microbial genomes, as well as $\sim 600$ novel plasmids and viruses. Altogether 5,002 results by 76 program versions were analyzed, representing a $22 x$ increase in results.

Substantial improvements were seen in metagenome assembly, some due to using long-read data. The presence of related strains still was challenging for assembly and genome binning, as was assembly quality for the latter. Taxon profilers demonstrated a marked maturation, with taxon profilers and binners excelling at higher bacterial taxonomic ranks, but underperforming for viruses and archaea. Assessment of clinical pathogen detection techniques revealed a need to improve reproducibility. Analysis of program runtimes and memory usage identified highly efficient programs, including some top performers with other metrics. The CAMI II results identify current challenges, but also guide researchers in selecting methods for specific analyses. 


\section{Introduction}

Over the last two decades, advances in metagenomic techniques have vastly increased our knowledge of the microbial world and intensified development of data analysis techniques $^{1-5}$. This created a need for unbiased and comprehensive performance assessment of these methods, to identify best practices as well as open challenges in the field $^{6-13}$. CAMI, the Initiative for the Critical Assessment of Metagenome Interpretation, is a community-driven effort that addresses this need, by offering comprehensive benchmarking challenges and datasets representing common experimental settings, data generation techniques, and environments in microbiome research. In addition to its open and collaborative nature, data FAIRness and reproducibility are key defining principles ${ }^{14}$.

The first CAMI challenge delivered insights into the performances of metagenome assembly, genome and taxonomic binning and profiling programs across multiple complex benchmark datasets, including unpublished genomes across a range of evolutionary divergences and of poorly categorized taxonomic groups, such as viruses. The robustness and high accuracy observed for genome binning programs in the absence of strain diversity supported their application to large-scale data from various environments, recovering thousands of metagenome-assembled genomes ${ }^{15-17}$ (MAGs), and intensified efforts in advancing strain-resolved assembly and binning techniques. We here describe the results of the second round of CAMI challenges ${ }^{18}$, in which we assessed program performances and progress on even larger and more complex datasets, additionally including long-read data and assessment of key performance metrics such as runtime and memory use.

\section{Results}

We created three comprehensive metagenome benchmark datasets representing a marine environment, a plant-associated environment that included fungal genomes and host plant material, as well as a very high strain diversity environment ("strain madness"). Datasets included both long and short-read data and were sampled from 1,680 microbial genomes and 599 circular elements of viruses and plasmids (Methods, Supplementary 
Table 1). Of these, 772 genomes and all circular elements were newly sequenced and distinct from taxa represented in public genome sequence collections (novel genomes), and the remainder were high-quality public genomes. Genomes were classified as "unique", if they had an Average Nucleotide Identity (ANI) of less than $95 \%$ to any other genome, or "common", if there were genomes with an ANI $\geq 95 \%$ in the benchmark data, as in the first CAMI challenge ${ }^{6}$. Overall, 901 genomes were unique: 474 for marine, 414 for plant-associated, and 13 for the strain madness data; and 779 were common: 303 for marine, 81 for plant-associated, and 395 for strain madness. In addition, a pathogen detection challenge was offered, based on a clinical metagenome sample from a critically ill patient with an unknown infection. Challenge participants were encouraged to submit reproducible results by providing executable software with exact parameter settings and reference databases used. Over all challenges, 5,002 results for 76 programs were received from 30 teams (Supplementary Table 2).

\section{Assembly challenge}

Sequence assembly is a key component of metagenome analysis, with assemblies being subsequently used to recover genome and taxon bins. Assembly quality degrades for genomes with low evolutionary divergences, resulting in consensus or highly fragmented assemblies $^{19,20}$. Due to the relevance of strain-resolved assemblies for understanding microbial communities ${ }^{21-23}$, we assessed methods' abilities to assemble strain-resolved genomes, together with the value of long and short-read data for assembly (Methods).

\section{Overall trends}

We evaluated 155 submissions (Supplementary Table 2) for 20 assembler versions: ASTAR workflow (hybrid, contigs, and scaffolds), ABySS ${ }^{24}$ (short read, v.2.1.5), (meta)Flye ${ }^{25}$ (long read, v.2.4.1, v.2.8, v.2.8.1), (Meta)HipMer ${ }^{26-28}$ (short read, v.1.0, v.1.2.2, v.2.0, Metagenome, cgraph, cgraph-ono), GATB ${ }^{29,30}$ workflow (hybrid, v.1.0), MEGAHIT ${ }^{31}$ (short read, v.1.1.2, v.1.1.4-2, v.1.2.7), Metahit_LINKS, Atlas ${ }^{32}$ (short read/hybrid single samples, v.2.1.0), (meta)SPAdes ${ }^{33,34}$ (short read/hybrid, v.3.13.0, v.3.13.1, v.3.14-dev), OPERA-MS ${ }^{35}$ (hybrid, v.0.8.3, v.0.9), and Ray Meta ${ }^{36}$ (short read, v.2.3.1), including some with multiple settings and different data preprocessing options. 
In addition, we created gold standard co- and single sample assemblies as in ${ }^{19}$, which included all regions covered by at least one read in the community-specific genome collections (i.e., marine, strain madness, plant-associated). The three gold standards of short, long, and hybrid marine data comprise $2.59 \mathrm{~Gb}, 2.60 \mathrm{~Gb}$, and $2.79 \mathrm{~Gb}$ of assembled sequences, respectively, while the strain madness short, long, and hybrid gold standards consist of $1.45 \mathrm{~Gb}$ each.

Assemblies were evaluated with MetaQUAST v.5.1.0 $\mathrm{rc}^{37}$, which was adapted for the evaluation of strain-resolved assembly (Supplementary text). To test the ability of the assemblers to generate near-complete strain-resolved genomes, we determined strain recall and precision, similar to ${ }^{38}$ (Supplementary Table 3). Strain recall measures how many genomes are recovered with high genome fraction and few mismatches (mm). Complementary to recall, strain precision assesses how accurately reference genomes are recovered, based on the fraction of correctly assembled high-quality, near-complete genomes (>90\% genome fraction, $<0.1 \% \mathrm{~mm}$ ) divided by the overall number of assembled, near-complete genomes (>90\% genome fraction). To facilitate comparisons, we ranked assemblies produced with different versions and parameter settings for a particular method based on key metrics (Methods) and chose the highest-ranking assembly as the representative (Fig. 1).

Of these, short-read assemblers achieved genome fractions of up to $10.4 \%$ on the complete strain madness and $41.1 \%$ on marine data, both by MEGAHIT. The gold standard reported $90.8 \%$ and $76.9 \%$, respectively (Fig. 1a, Supplementary Table 3). ASTAR excelled in terms of genome fraction on both data sets, but created more misassemblies and $\mathrm{mm}$ than others. HipMer had the fewest $\mathrm{mm}$ per $100 \mathrm{~kb}$ on the marine data set with 96 and GATB on the strain madness with 98 (Fig. 1b). The best hybrid assembler, A-STAR, improved the genome fraction to $44.1 \%$ on the marine dataset, at a cost of $773 \mathrm{~mm} / 100 \mathrm{~kb}$. The fewest mismatches (173) for hybrid assemblers were introduced by GATB. ABySS created the fewest misassemblies for the marine and GATB for the strain madness data (Fig. 1c). The most contiguous assemblies were provided by the hybrid assembler OPERA-MS for the marine data (Fig. 1d), with an average NGA50 


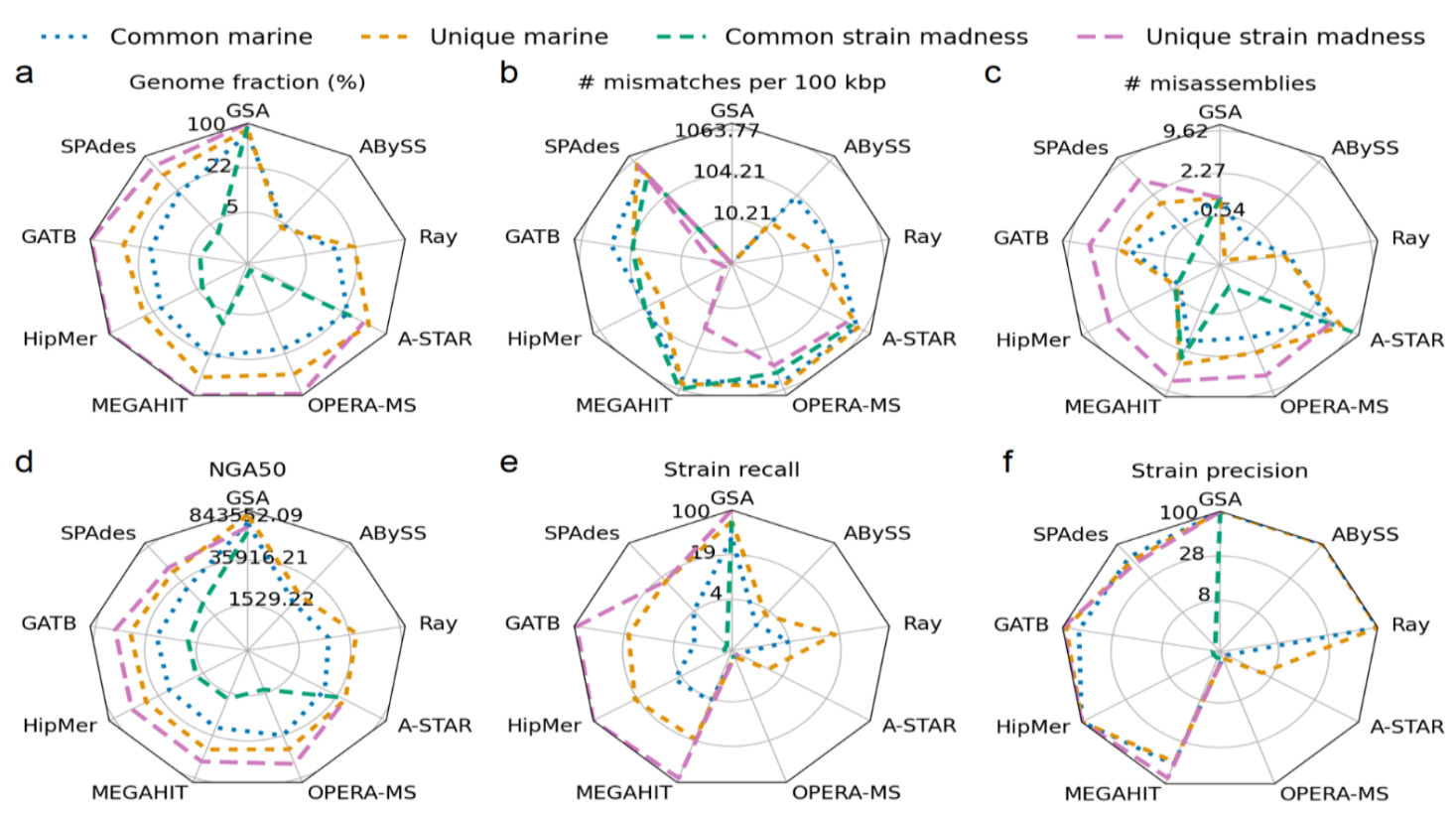

g Genome groups - Circular elements - Common (ANI $\geq 95 \%)$ - Unique (ANI <95\%)
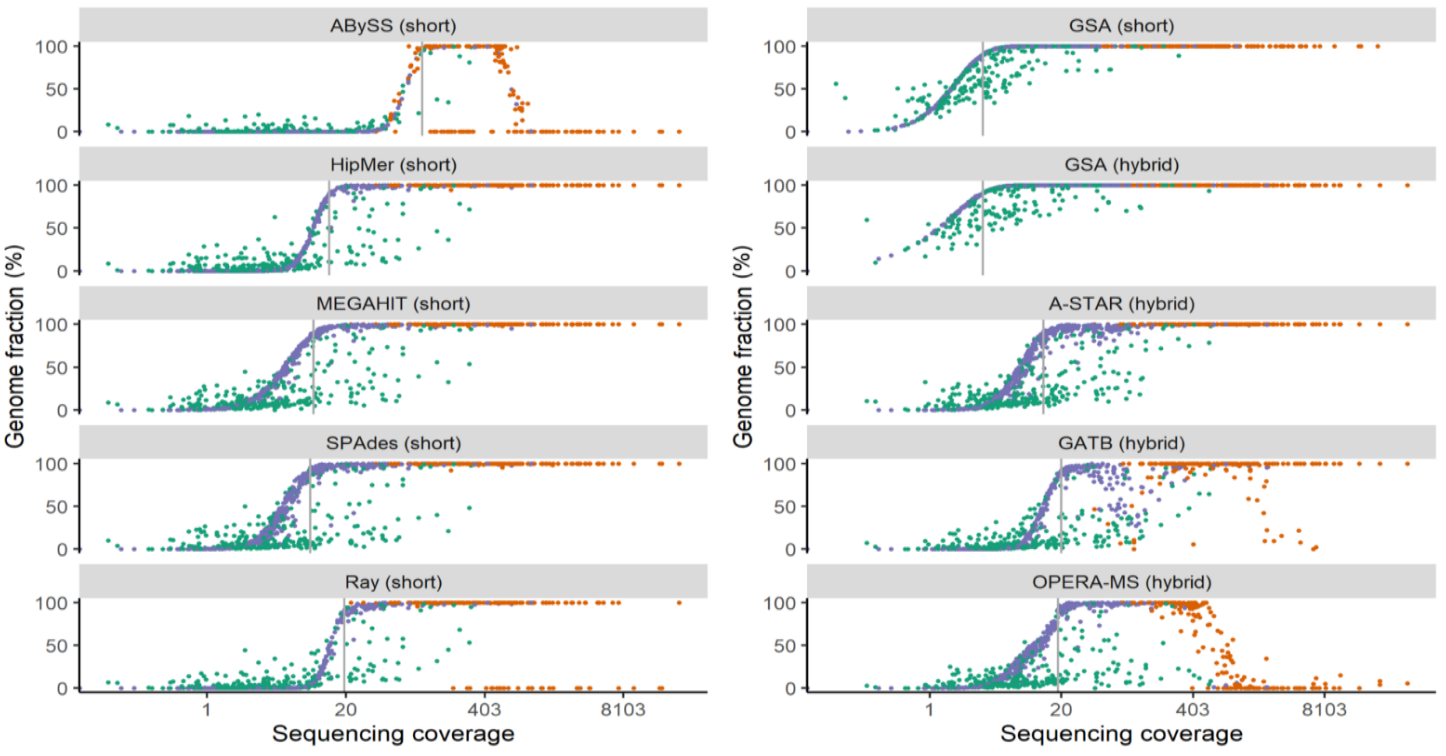

Fig. 1: Radar plots of genome fraction (a), mismatches per $100 \mathrm{~kb}$ (b), misassemblies (c), NGA50 (d), strain recall (e), and precision (f) for assemblers on marine and strain madness data. For methods with multiple evaluated versions, the best ranked version on the marine data is shown. Absolute values for metrics are log scaled. Lines indicate different subsets of genomes analyzed, and the value of the gold standard assemblies (GSA) indicates the upper bound for a metric. The metrics are shown for both unique and common strain genomes. g, Genome recovery fraction versus genome sequencing depth (coverage) on the marine dataset. Blue indicates unique genomes ( $<95 \% \mathrm{ANI})$, green common genomes (ANI $\geq 95 \%$ ), and orange high-copy circular elements. Grey lines indicate the coverage at which the first genome is recovered with $\geq 90 \%$ genome fraction. 
of 28,244 across genomes, in comparison to 682,777 for the gold standard. The SPAdes hybrid submission had a higher NGA50 of 43,014, but it was not the best scoring SPAdes submission. A-STAR had the highest contiguity for the strain madness data $(13,008 \mathrm{vs.}$ 155,979 for gold standard). For short-read assembly, MEGAHIT had the highest contiguity on the marine (NGA50 of 26,599) and on the strain madness data (NGA50 of $4,793)$. Notably, differently from what we observed for the plant-associated long-read data (Supplementary Fig. 1), Flye performed less well than other assemblers across most metrics on the marine data, likely due to use of different versions or parameter settings (Supplementary Table 2).

HipMer ranked first across metrics on the marine data, as it produced few $\mathrm{mm}$ combined with a comparably high genome fraction and NGA50. On the strain madness data, GATB ranked best, with HipMer in second place. On the plant-associated dataset, HipMer again performed best across metrics, followed by Flye, whereby both outperformed the other short-read assemblers on this dataset in most metrics (Supplementary Fig. 1).

For several assemblers, preprocessing the data had a substantial impact on assembly quality (Supplementary Tables 2 and 3). In particular, using read quality trimming or error correction software, such as trimmomatic ${ }^{39}$, DUK ${ }^{40}$, Fastp ${ }^{41}$, or Bayeshammer ${ }^{42}$, improved assembly quality. Genome coverage was also a key factor for assembly quality (Fig. 1g). While both gold standards for short and hybrid assemblies contained genomes with more than $90 \%$ genome fraction starting at a coverage of $3.3 x$, the best assembler for low coverage genomes on the marine data set was SPAdes, recovering unique genomes starting at 9.2x. MEGAHIT, A-STAR, and HipMer required 10x, 13.2x, and 13.9x coverage, while Ray Meta recovered almost complete genomes from a coverage of 19.5x. Several assemblers reconstructed high copy circular elements well, with HipMer, MEGAHIT, SPAdes, and A-STAR reconstructing all of them (Fig. 1g). In comparison to well-performing software assessed in the first CAMI challenge, A-STAR substantially improved in genome fraction for the strain madness data by $20 \%$ (almost 3 times the genome fraction in relative terms) relative to MEGAHIT. HipMer introduced the fewest mismatches on the marine $(67 \mathrm{~mm} / 100 \mathrm{~kb})$ data. This was $30 \%$ less than Ray Meta, the best performing method also participating in CAMI 1. OPERA-MS improved on MEGAHIT 
in terms of NGA50 by 1,645 (6\%), though using twice as much (long and short-read) data. SPAdes, which was not assessed in the first challenge, consistently was among the top submissions for most metrics.

\section{Closely related genomes}

The first CAMI challenge revealed substantial differences in assembly quality between unique and common strain genomes ${ }^{6}$. We further focused on this by providing a dataset consisting almost entirely of common strain genomes. The upper bound for strain recall was $54.9 \%$, as provided by the marine gold standard assembly, and $67.4 \%$ for the strain madness one (Fig. 1e). Strain recall varied little across several evaluated threshold settings for genome fraction $(>90 \%,>75 \%)$ and mismatches $(<0.1 \% \mathrm{~mm} / \mathrm{kb} ;<0.5 \%$ $\mathrm{mm} / \mathrm{kb}$ ). Therefore, we set $>90 \%$ genome fraction and $<0.1 \% \mathrm{~mm} / \mathrm{kb}$ as thresholds.

Overall, GATB ranked best across metrics on strain madness data and the common strain madness genomes, while HipMer ranked best on marine data and common marine genomes (Supplementary Table 3). HipMer had the highest strain recall $(14.4 \%$ on marine, $3.2 \%$ on strain madness), similar or even better than the best hybrid assembler, GATB (10.8\% on marine, $2.9 \%$ on strain madness). Multiple assemblers - ABySS, HipMer, and Ray Meta on marine and GATB on strain madness - achieved 100\% precision (Fig. 1f). For strain madness common genomes, A-STAR recovered the most, with $1.5 \%$ recall $(<0.5 \% \mathrm{~mm},>75 \%$ genome fraction) and $23.1 \%$ strain precision. HipMer recovered a lower genome fraction (4.1\% versus $30.4 \%$ for A-STAR), but also created fewer misassemblies per genome $(0.5)$ and mismatches $(0.1 \%)$, resolving fewer, but higher quality strain genomes with high precision $(0.8 \%$ strain recall, $100 \%$ strain precision). A major difference between common genomes in the two datasets is that, in the strain madness data, virtually all genomes form a single cluster with $>95 \%$ ANI, while the marine data includes multiple clusters with a few closely related genomes each. Accordingly, strain recall was higher for common marine than common strain madness genomes (Supplementary Table 4). On marine common genomes, SPAdes had the highest strain recall $(8.7 \%)$ and $96.7 \%$ strain precision, followed by A-STAR $(7.5 \%$ recall, 
$69.4 \%$ precision). A-STAR (26.7\%) and MEGAHIT (24.6\%) achieved the highest genome fractions.

Across metrics, unique genome assemblies were superior to common genome ones (Fig. 1, Supplementary Table 4 ), for marine genomes by $15.7 \%$ in strain recall, $22.7 \%$ genome fraction, 3-fold NGA50, except in strain precision (-1.2\%), on average (Supplementary Table 5), resulting in substantially more complete, higher quality, and less fragmented assemblies. A-STAR provided the most complete assemblies (55.3\% genome fraction), the HipMer assembly had the highest strain recall (20.4\%), HipMer, ABySS, Flye, and Ray Meta the highest strain precision (100\%) and OPERA-MS an exceptional average NGA50 $(187,083,75 \%$ of the gold standard NGA50). HipMer ranked best across all metrics, with $100 \%$ strain recall and precision, $98.5 \%$ genome fraction, and the fewest mismatches $(0.001 \%)$. Unique strain madness genomes were recovered best, with on average $81.4 \%$ strain recall, $89.4 \%$ strain precision, $86.4 \%$ genome fraction, and an NGA50 of 120,771 (Supplementary Table 6).

\section{Difficult to assemble regions}

As the marine data also include high quality public genomes, assembly performance can be assessed for particularly difficult to assemble genomic regions, such as repeats or highly conserved elements (e.g., 16S). To assess recovery of complex regions, we selected 50 unique, public genomes present as a single contig in the gold standard and with annotated $16 \mathrm{~S}$ sequences. We mapped assembly submissions to these $16 \mathrm{~S}$ sequences and measured their completeness and gap-compressed divergence (Supplementary Fig. 2). A-STAR partially recovered 102 (78\%) of 131 16S gold standard sequences. The hybrid assemblers GATB (mean recovered gene fraction 60.1\%) and OPERA-MS (mean 47.1\%) recovered the most complete $16 \mathrm{~S}$ sequences. The mean fraction of genes recovered by short-read assemblers spanned between 29.6\% (HipMer) and 36.9\% (MEGAHIT) and were very accurate for ABySS and HipMer (<1\% divergence). Average assembly quality was better for public than for novel genomes in key metrics, such as genome fraction and NGA50 (Supplementary Fig. 3). 


\section{Single versus co-assembly}

For multi-sample metagenome datasets, there are two assembly strategies: pooling samples (coassembly) and single-sample assembly ${ }^{16,28,43}$. We evaluated the assembly quality of both strategies for genomes that were spiked into the plant-associated data with specific coverages (Supplementary Table_8) across submitted results for five assemblers (Supplementary Fig. 4). Two genomes were unique with $8 x$ coverage across the pooled samples, distributed into 16 samples or one sample, respectively. For the genome split across 16 samples, only HipMer recovered it from the pooled samples, while the genome present in only one sample was reconstructed well by all assemblers from single and pooled samples. For genomes unique to a single sample, but common in pooled samples (LjRoot109, LjRoot170), HipMer showed a better performance on the single samples, while OPERA-MS generally performed better on the pooled samples (Supplementary Fig. 4 ), and other assemblers traded a higher genome fraction against more mismatches. Thus, co-assembly could improve assembly for OPERA-MS in general and for short-read assemblers on low coverage genomes without expected strain diversity across samples. For HipMer, single-sample assembly might be preferable, if coverage is sufficient and closely related strains are expected.

\section{Genome binning challenge}

Genome binners group contigs or reads together to recover genome bins from metagenome data. We evaluated 95 results for 18 binning software versions on short read-assemblies: 22 for the strain madness gold standard assemblies (GSA), 17 for the strain madness MEGAHIT assembly (MA), 19 for marine MA, 15 for the marine GSA, 12 for the plant-associated GSA, and 10 for the plant-associated MA (Supplementary Tables 9-15, Supplementary Table 2 for software availability, versions, and parameters). In addition, 7 results on the plant-associated hybrid assemblies were evaluated. Methods included well performing ones from the first CAMI challenge and popular software, such as MetaBAT 44,45 (v.2.15-5, v.2.13-33, v.0.25.4), $\operatorname{MaxBin}^{46}$ (v.2.2.7, v.2.0.2) and $\operatorname{CONCOCT}^{47}$ (v.1.1.0, v.0.4.1), as well as Autometa ${ }^{48}$ (v.cami2), LSHVec ${ }^{49}$ (v.cami2), MetaBinner (v.1.3, v.1.2, v.1.1, v.1.0), UltraBinner (v.1.0), MetaWRAP50 (v.1.2.3), 
SolidBin ${ }^{51}$ (v.1.3), and $\operatorname{Vamb}^{52}$ (v.3.0.1, v.fa045c0). While for GSA contigs, the ground truth genome assignment is known, for the MA, we considered the ground truth for a contig to be the best matching genomes identified using MetaQUAST v5.0.2 with default settings. We assessed the average purity of bins and completeness of genomes (and their summary using the F1-score), the number of high-quality genomes recovered, as well as the Adjusted Rand Index (ARI) for the binned data, using AMBER v.2.0.353 (Methods). The ARI, in combination with the fraction of binned data, quantifies binning performance for the overall dataset.

The performance of genome binners varied widely across metrics, software versions, datasets, and assembly type (Fig. 2), while parameters affected performance mostly by less than $3 \%$. For the marine GSA, average bin purity was $81.3 \pm 2.3 \%$ and genome completeness was $36.9 \pm 4.0 \%$ (Fig. 2a,b, Supplementary Table 9). For the marine MA, average bin purity $(78.3 \pm 2.6 \%)$ was similar, while average completeness was only $21.2 \pm 1.6 \%$ (Fig. 2a,c, Supplementary Table 10), due to many short contigs with $1.5-2 \mathrm{~kb}$, which most binners did not attempt to bin (Supplementary Fig. 5). For the strain madness GSA, average purity and completeness decreased, by $20.1 \%$ to $61.2 \pm 2.3 \%$, and by $18.7 \%$ to $18.2 \pm 2.2 \%$, respectively, relative to the marine GSA (Fig. 2a,d, Supplementary Table 11). While the average purity on the strain madness MA $(65.3 \pm 4.0 \%)$ and GSA were similar, the average completeness dropped further to $5.2 \pm 0.6 \%$, again due to a larger fraction of short contigs not binned (Fig. 2a,e, Supplementary Table 12). For the plant-associated GSA, purity was almost as high as for marine $(78.2 \% \pm 4.5$; Fig. 2a,f, Supplementary Table 13), but bin completeness decreased relative to other GSAs $(13.9 \pm 1.4 \%)$, due to poor recovery of the mostly low abundant, large, fungal genomes. Notably, the $A$. thaliana host genome (5.6x coverage) as well as fungi with more than $8 x$ coverage were binned with much higher completeness and purity than genomes with lower coverage (Supplementary Fig. 6). Binning of the hybrid assembly further increased average purity to $85.1 \pm 6.3 \%$, while completeness remained similar $(11.9 \pm 2.1 \%$, Supplementary Table 14). For the plant-associated MA, average purity $(83 \pm 3.3 \%)$ and

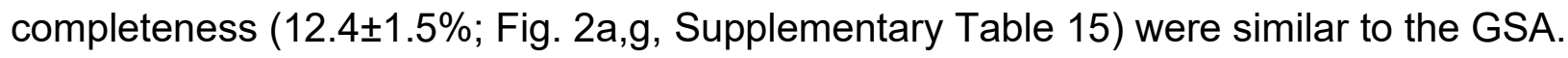




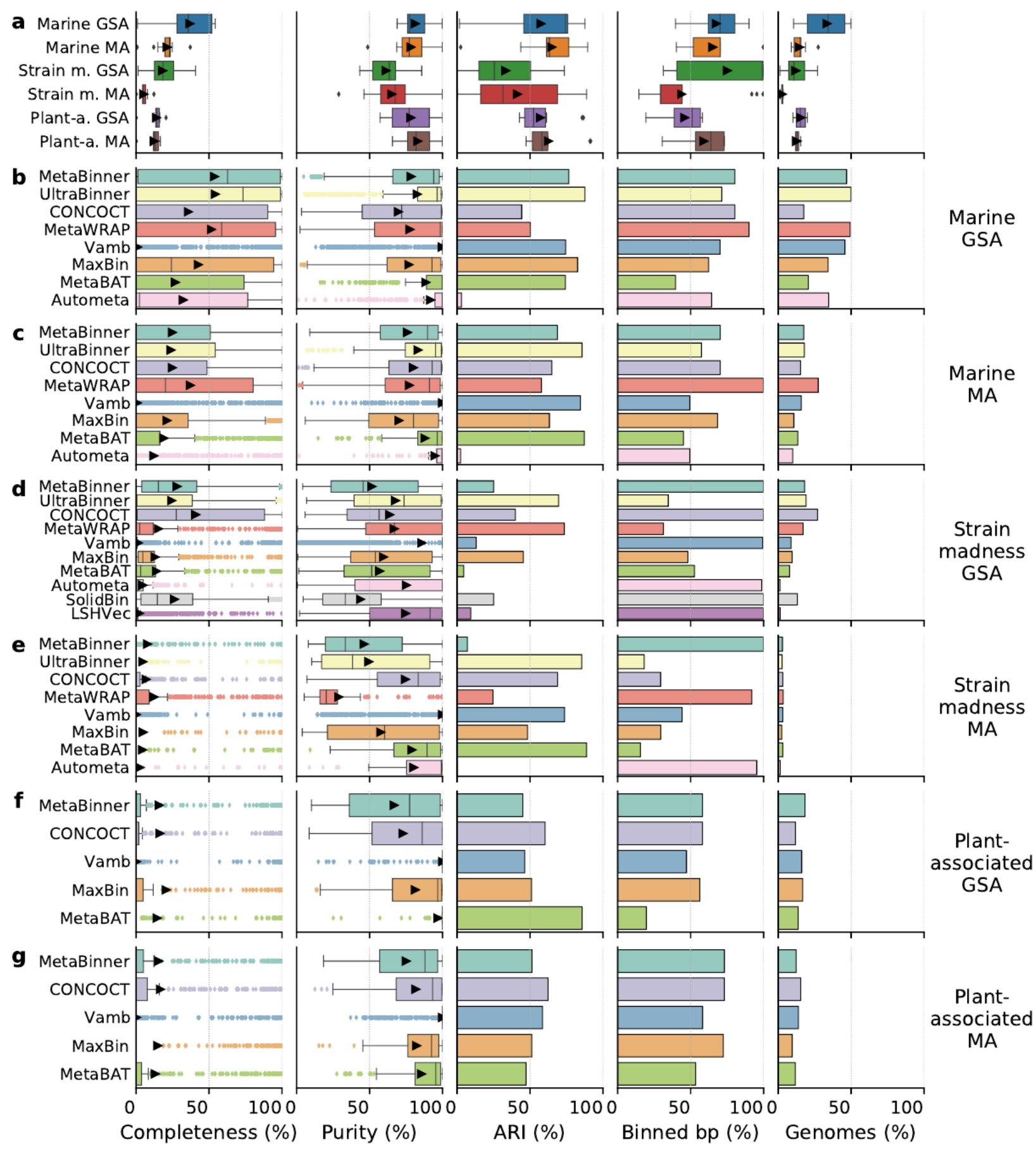

Fig. 2: Performance of genome binners on short-read assemblies (GSA: gold standard, MA: MEGAHIT) of the marine, strain madness, and plant-associated data. a, Boxplots of average completeness, purity, Adjusted Rand Index (ARI), percentage of binned bp, and fraction of genomes recovered with moderate or higher quality ( $>50 \%$ completeness, $<10 \%$ contamination) across methods from each dataset (Methods). Arrows indicate the average. $\mathbf{b}-\mathbf{g}$, Boxplots of completeness per genome and purity per bin, and bar charts of ARI, binned bp, and moderate or higher quality genomes recovered, by method, for each dataset. The submission with the highest F1-score per method on a dataset is shown (Supplementary Tables 9-15). 
To quantitatively assess binners across gold standard and real assemblies for the three datasets, we ranked the best submissions (Supplementary Tables 16-19) across metrics (Methods). For marine and strain madness, the best trade-off performances were given by CONCOCT and MetaBinner for MAs, UltraBinner for GSAs, and MetaBinner overall. CONCOCT also performed best on plant-associated assemblies. UltraBinner had the best completeness on the marine GSA, CONCOCT on the strain madness GSA and plant-associated MA, MetaWRAP on marine and strain madness MAs, and MaxBin on the plant-associated GSA. Vamb had the best purity in all settings, while UltraBinner had the best ARI for the marine GSA, MetaWRAP for the strain madness GSA, and MetaBAT for MAs and all plant-associated assemblies. MetaWRAP and MetaBinner assigned the most for the marine and plant-associated assemblies, respectively, and many methods assigned all strain madness contigs, though with low ARI (Fig. 2b-g). UltraBinner recovered the most high-quality genomes from the marine GSA, MetaWRAP from the marine MA, CONCOCT from strain madness assemblies and plant-associated GSA, and MetaBinner from the plant-associated GSA and hybrid assemblies (Fig. 2, Supplementary Table 20). For plasmids and other high copy circular elements, Vamb performed best, with an F1-score of $70.8 \%, 54.8 \%$ completeness, and $100 \%$ purity, while the next best method, MetaWRAP, had an F1-score of 12.7\% (Supplementary Table 21).

\section{Effect of strain diversity}

For both marine and strain madness GSAs, binning of unique strains was substantially better than for common strains (Supplementary Fig. 7, Supplementary Tables 9 and 11). Differences were more pronounced on strain madness, for which unique strain bin purity was particularly high $(97.9 \pm 0.4 \%)$. The best ranking across metrics and these four data partitions was obtained by UltraBinner for unique genomes and overall, as well as CONCOCT for common strains (Supplementary Table 22). UltraBinner had the highest completeness on unique strains, while CONCOCT ranked best for common strains and across all partitions together. Vamb ranked first by purity in all settings, UltraBinner by $\mathrm{ARI}$, and MetaBinner by most assigned. Due to the dominance of unique strains in the marine and common strains in the strain madness dataset, the best binners in the 
respective data were the same as for the entire datasets (Supplementary Tables 9 and 11) and performances similar for most metrics.

\section{Taxonomic binning challenge}

Taxonomic binners group sequences into bins labelled with a taxonomic identifier. For taxonomic binning, we evaluated 547 results for nine methods and versions: LSHVec v.cami2 ${ }^{49}$, PhyloPythiaS+ v.1.4 ${ }^{54}$, Kraken v.2.0.8-beta55 and v.0.10.5-beta (cami1), DIAMOND v.0.9.2856, MEGAN v.6.15.257, Ganon v.0.1.4 and v.0.3.158, and NBC++59. Of these, 75 were for the marine, 405 for strain madness, and 67 for plant-associated data, on either reads or gold standard assemblies (Supplementary Tables 2). We assessed the average purity and completeness of bins and the accuracy per sample at different taxonomic ranks (Methods).

On the marine data, average taxon bin completeness across ranks was $63 \%$, average purity $40.3 \%$, and accuracy per sample bp 74.9\% (Fig. 3a, Supplementary Table 23). On the strain madness data, accuracy was similar (76.9\%; Fig. 3b, Supplementary Table 24), while completeness was $\sim 10 \%$ higher and purity lower by that much. On the plantassociated data, purity was between those of the first two datasets (35.\%), but completeness and accuracy were lower (44.2\% and 50.8\%, respectively; Fig. 3c, Supplementary Table 25). For all datasets, performances declined at lower taxonomic ranks, most notably from genus to species rank by $22.2 \%$ in completeness, $9.7 \%$ in purity, and $18.5 \%$ in accuracy, on average.

Across all datasets, MEGAN on contigs ranked first across metrics and all ranks (Supplementary Table 26), closely followed by Kraken v.2.0.8-beta on contigs and then by Ganon on short reads. Kraken v.2.0.8-beta on contigs was the best for genus and species, and on marine data in completeness and accuracy (89.4\%, 96.9\%; Supplementary Table 23 and 27). Due to the presence of public genomes, Kraken's completeness on marine data was much higher than in the first challenge, particularly at species and genus rank (average of $84.6 \%$ and $91.5 \%$, respectively, compared to $50 \%$ and $5 \%$ ), while purity remained similar. MEGAN on contigs ranked highest for taxon bin purity on the marine and plant-associated data $(90.7 \%, 87.1 \%$; Supplementary Tables 
- Av. completeness ( $-=1 \%$ filtered)

a

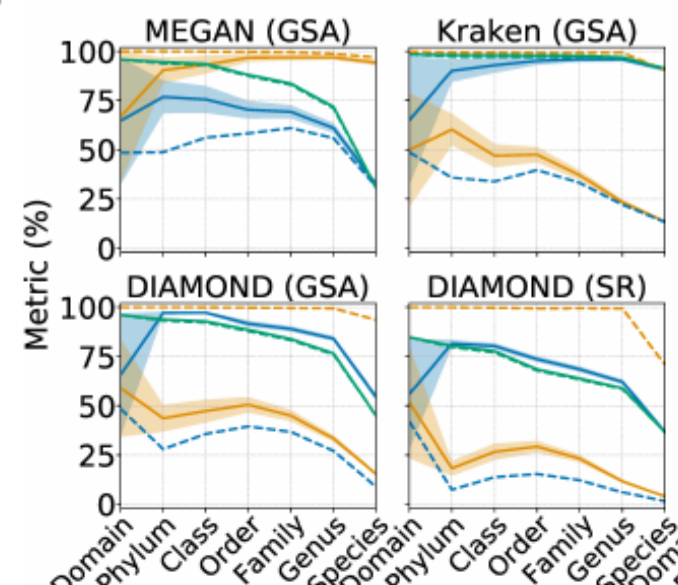

- Av. purity (- = $1 \%$ filtered)

- Accuracy (- - 1\% filtered)

Marine

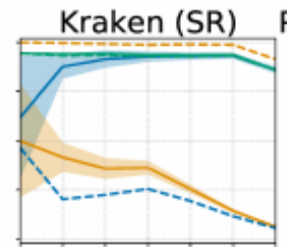

$\mathrm{NBC}++(\mathrm{SR})$
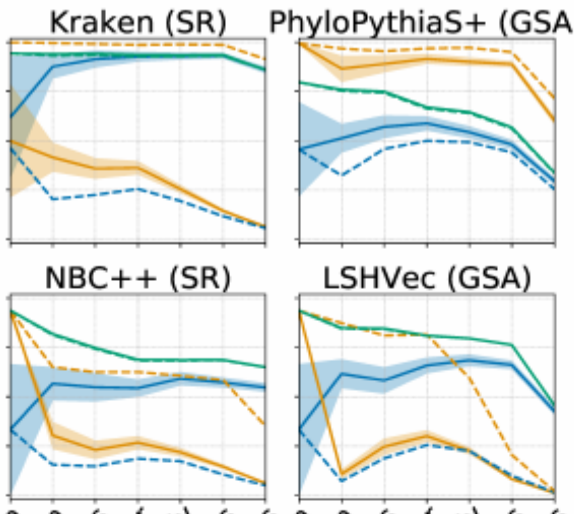

LSHVec (GSA)
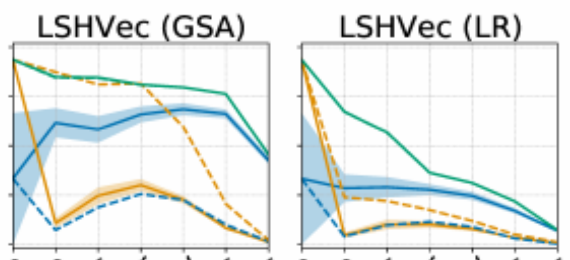

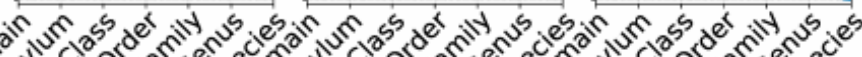

\section{Strain madness}

b

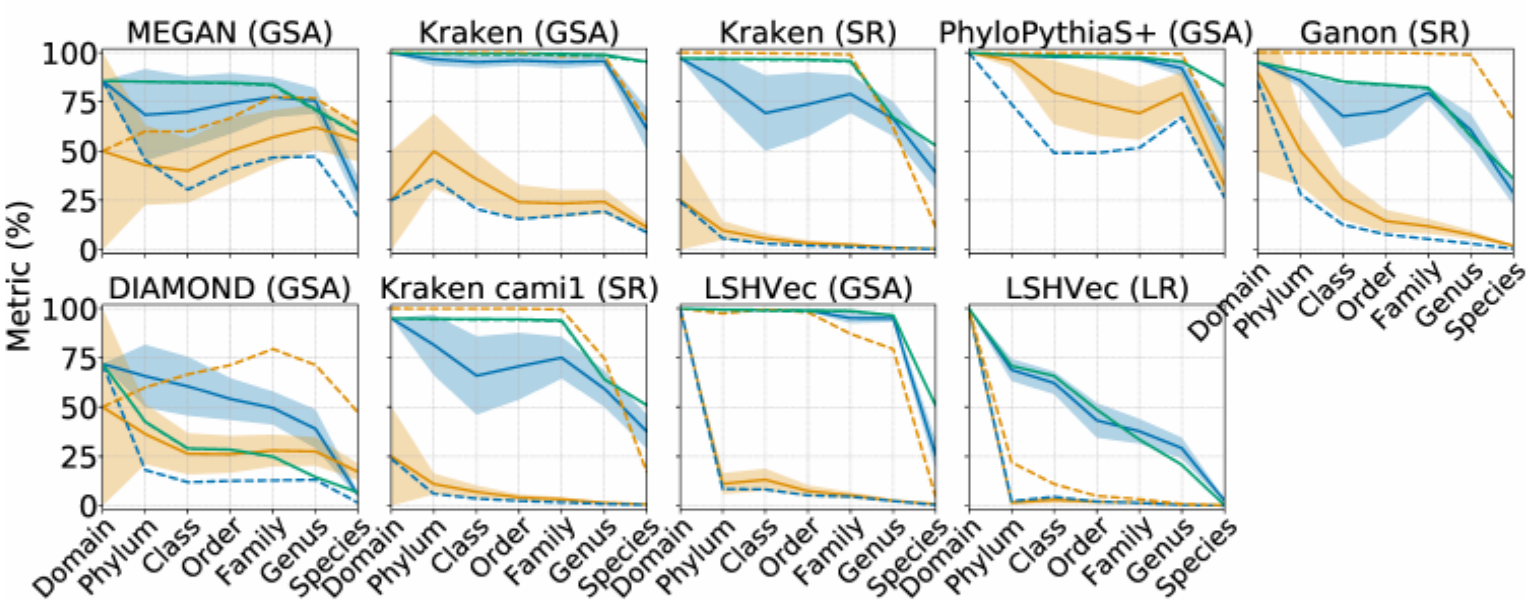

C

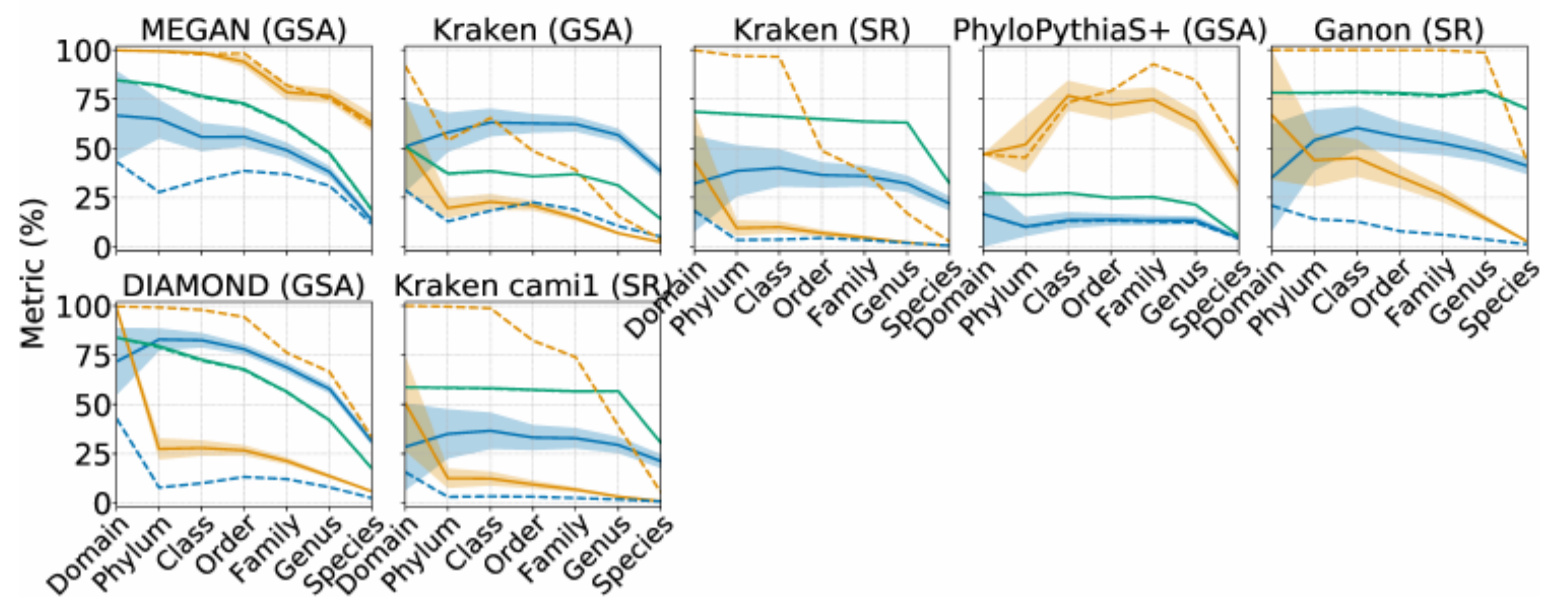

Fig. 3: Taxonomic binning performance across ranks for the marine (a), strain madness (b), and plant-associated datasets (c). Metrics were computed over unfiltered (solid lines) and 1\%-filtered (i.e., without the 1\% smallest bins in bp, dashed lines) predicted bins of short reads (SR), long reads (LR), and contigs of the gold standard assembly (GSA). Shaded bands show the standard error across bins. 
23, 25, 27, and 28). PhyloPythiaS+ ranked best for the strain madness data across metrics, as well as in completeness (90.5\%) and purity (75.8\%) across ranks (Supplementary Tables 24 and 29). DIAMOND on contigs ranked best for completeness $(67.6 \%)$ and Ganon on short reads for accuracy $(77.1 \%)$ on the plant-associated data.

Filtering of the $1 \%$ smallest predicted bins per taxonomic level is a popular postprocessing approach. On all datasets, filtering increased average purity to above $71 \%$ and reduced completeness, to $\sim 24 \%$ on marine and strain madness and $13.4 \%$ on plantassociated data (Supplementary Table 23-25). Accuracy was not much affected, as large bins contribute more to this metric. Kraken on contigs still ranked first in filtered accuracy and MEGAN across all filtered metrics (Supplementary Table 26). MEGAN on contigs and Ganon on short reads profited the most from filtering, ranking first in filtered completeness and purity, respectively, across all datasets and taxonomic levels.

\section{Taxonomic binning of divergent genomes}

To investigate the effect of increasing divergence between query and reference sequences for reference-based taxonomic binners, we categorized genomes by their distances to public genomes (Supplementary Fig. 8, Supplementary Tables 30 and 31). Sequences of known marine strains were assigned particularly well at the species rank by Kraken (accuracy, completeness, and filtered purity above 93\%) and MEGAN (91\% purity, 33\% completeness and accuracy). Kraken also best classified new strain sequences at species level, though with less completeness and accuracy for the marine data (68\% and $80 \%$, respectively). It also had the best accuracy and completeness across ranks, but low unfiltered purity. For the strain madness data, PhyloPythiaS+ performed similarly well up to genus level, and best assigned new species at genus level (93\% accuracy and completeness, and $75 \%$ filtered purity). Only DIAMOND classified viral contigs, though with low purity (50\%) and completeness and accuracy (both $3 \%$ ), and no method classified sequences as plasmids. 


\section{Taxonomic profiling challenge}

Taxonomic profilers quantify the presence and relative abundances of microbial community taxa from metagenome samples. This is in contrast to taxonomic sequence classification, which assigns taxon labels to individual sequences and results in taxonspecific sequence bins (and sequence abundance profiles), instead of taxonomic abundance profiles for entire samples or datasets ${ }^{60}$. We evaluated 4,195 profiling results (292 marine, 2,603 strain madness, and 1,300 plant-associated datasets), from 22 method versions (Supplementary Table 2) with the majority of the results originating from short-read samples, and a few from long-read samples, assemblies, or averages across samples. Performance was evaluated with OPAL v.1.0.1061 (Methods). The quality of predicted taxon profiles was determined based on completeness and purity of identified taxa, relative to the underlying ground truth, for individual ranks, while taxon abundance estimates were assessed using the L1 norm error for individual ranks, and the weighted Unifrac error across ranks. To assess alpha diversity of profiling results, the absolute difference between predicted and actual Shannon equitability index was determined (Methods).

\section{Taxon identification}

On the marine data, methods performed well until genus rank (average purity $70.4 \%$ and completeness $63.3 \%$ ), with a substantial drop at species level, to $44.4 \%$ purity and $47.1 \%$ completeness (Supplementary Fig. 9, Supplementary Tables 32 and 33). mOTUs v.2.5.162 had completeness and purity above $80 \%$ at genus and species ranks, and Centrifuge ${ }^{63}$ and MetaPhIAn 2.9.22 ${ }^{64,65}$ just at the genus rank (Fig. 4). Other methods with completeness above $80 \%$ at either rank were Bracken ${ }^{66}$, Centrifuge v.1.0.4 beta, MetaPhIAn v.2.9.22, and NBC++59, while CCMetagen ${ }^{67}$, DUDes $0.08^{68}$, LSHVec gsa ${ }^{49}$, Metalign $^{69}$, MetaPalette ${ }^{70}$, and MetaPhIAn cami1 had more than $80 \%$ purity. Filtering out the rarest $(1 \%)$ predicted taxa per rank decreased completeness by $\sim 22 \%$, while increasing precision by $\sim 11 \%$.

A similar trend was evident for the strain madness data, with methods performing well until the genus rank (average purity $52.1 \%$ and completeness $80.5 \%$ ), and a substantial 


\begin{tabular}{|c|c|c|c|c|}
\hline Bracken & DUDes cami1 & $\Delta$ Metalign & $\nabla$ MetaPhyler & NBC++ \\
\hline agen & FOCUS & MetaPalette & moTUs & TIPP \\
\hline ntrifuge & FOCUS cami1 & MetaPhIAn & 4 moTUs cami1 & TIPP cami1 \\
\hline DUDes & V LSHVec & MetaPhIAn cami1 & & \\
\hline
\end{tabular}

a

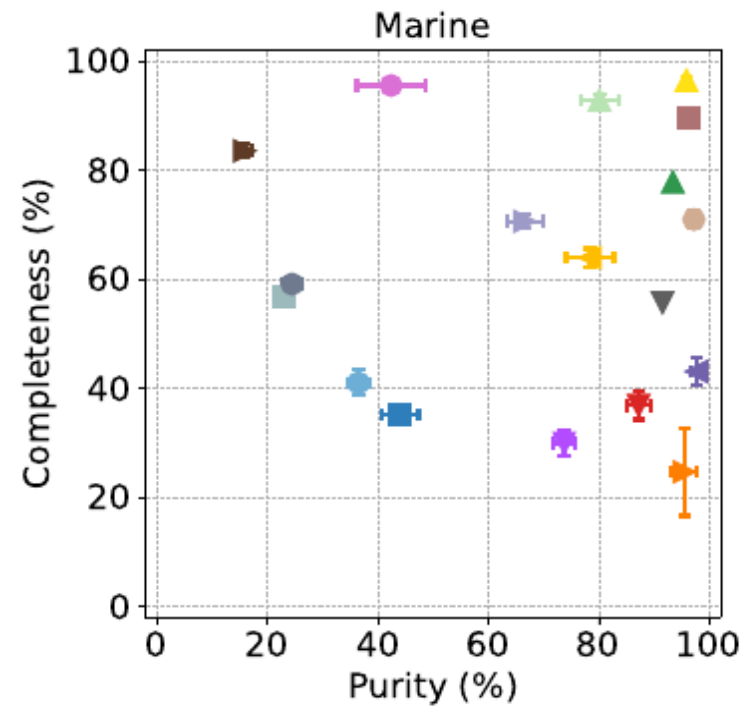

C

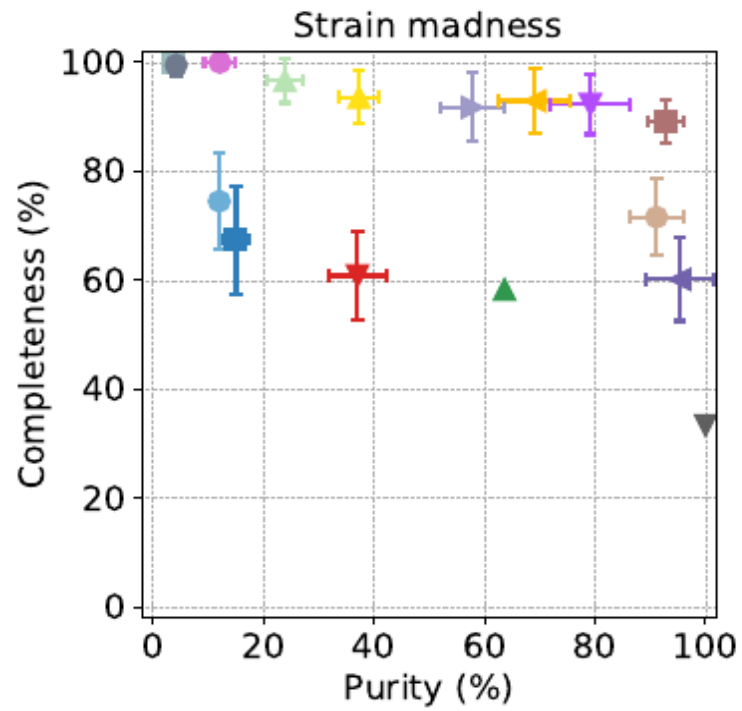

b

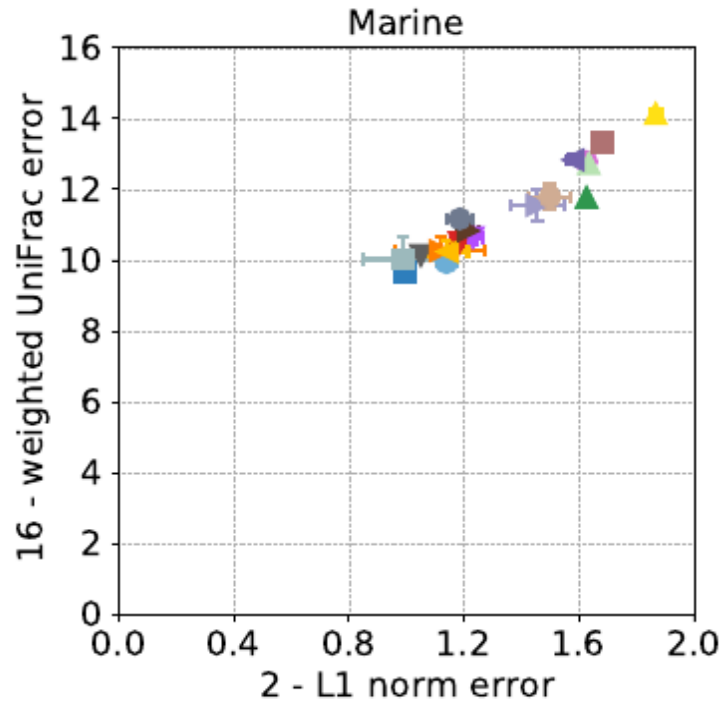

d

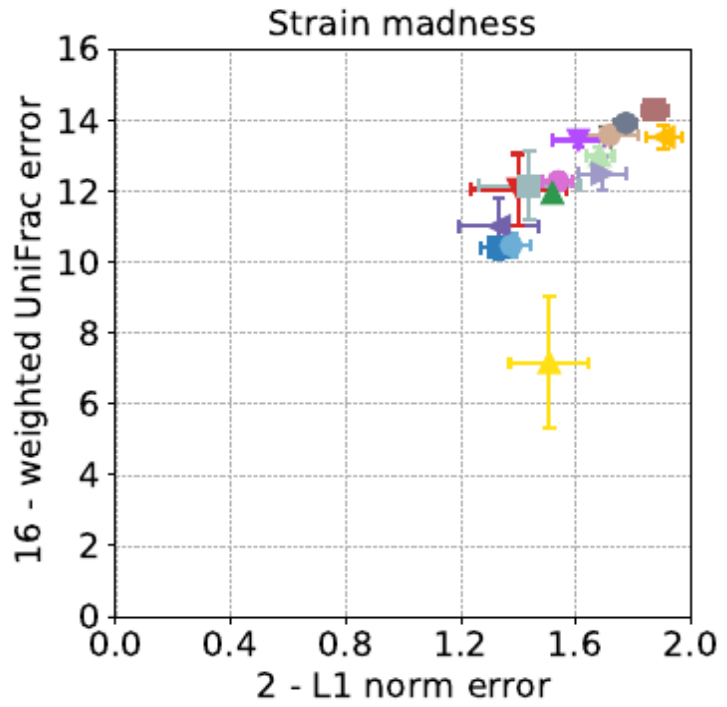

Fig. 4: Taxonomic profiling results for the marine $(a, b)$ and strain madness $(c, d)$ datasets at genus level. Results are shown for the overall best ranked submission per software version (Supplementary Tables 33 and 35 ). a, c, purity vs. completeness. b, d, upper bound of L1 norm minus actual L1 norm vs. upper bound of weighted UniFrac error minus actual weighted UniFrac error. Error bars show the standard deviation across samples. Metrics were determined using OPAL with default settings.

drop at species rank (average purity $22.3 \%$, completeness $43.9 \%$, Supplementary Tables 34 and 35 ). In comparison to the marine data, the average purity per method across ranks 
fell from $75.3 \%$ to $57.1 \%$, while completeness rose from $72 \%$ to $79 \%$. At the genus rank, particularly MetaPhIAn v.2.9.2 (89.2\% completeness, 92.8\% purity), MetaPhyler v.1.2571 (92.3\% completeness, $79.2 \%$ purity) and mOTUs of the first CAMI challenge $(92.9 \%$ completeness, $69.1 \%$ purity) performed well, but no method excelled at the species rank. DUDes v.0.08 and LSHVec gsa also had high purity, while Centrifuge v.1.0.4 beta, DUDes v.cami1, and TIPP v.4.3.10 and v.cami1 had high completeness.

Also for the plant-associated data, average purity and completeness decreased considerably from genus to species, from $62.9 \%$ to $31.7 \%$ and from $42.1 \%$ to $31.9 \%$, respectively (Supplementary Tables 36 and 37 ). The average purity across ranks was the highest among the datasets (77.8\%), while average completeness was the lowest $(49.7 \%)$, due to the many newly sequenced bacterial and fungal genomes (Methods), which most methods failed to detect. Bracken v.2.6 performed best for completeness across ranks (average 63.7\%) and "sourmash gather 3.3.2 k21"72 on short-reads for species (53.8\%). "Sourmash gather 3.3.2 k21" on Pacific Biosciences reads and MetaPhIAn v.3.0.7 on short reads had the highest purity across ranks $(94.7 \%, 94.67 \%)$, and the latter also for species (68.8\%).

\section{Relative abundances}

Abundances across ranks and submissions were on average predicted better for strain madness than marine data, which has less complexity above the strain level, with the L1 norm error improving from 0.44 to 0.3 , and average weighted UniFrac error from 4.65 to 3.79 (Supplementary Table 32, 34, 36). Abundance predictions were not as good on the plant-associated data and averaged 0.57 in L1 norm error and 5.16 in weighted UniFrac. On the marine data, mOTUs v.2.5.1 had the lowest L1 norm error at almost all levels and 0.12 on average, including the lowest at genus and species with 0.13 and 0.34 , respectively. It was followed by MetaPhIAn v.2.9.22, with 0.22 on average, and 0.32 and 0.39 at genus and species. Both methods also had the lowest weighted UniFrac error, followed by DUDEs v.0.08. On the strain madness data, mOTUs cami1 performed best in L1 norm error across ranks with 0.05 , and also at genus and species with 0.1 and 0.15 , followed by MetaPhIAn v.2.9.22 with 0.09 on average, and 0.12 and 0.23 at genus and species. The latter also had the lowest weighted UniFrac error, followed by TIPP v.cami1 
and mOTUs v.2.0.1. On the plant-associated data, Bracken v.2.6 had the lowest L1 norm error across ranks, with 0.36 on average, and at genus, with 0.34. "sourmash gather 3.3.2 k31" on short-reads had the lowest at species, with 0.55 . Both methods also had the lowest UniFrac error on this dataset. Several methods also accurately reconstructed the alpha diversity of samples using the Shannon equitability; best (0.03 or less absolute difference to gold standards) across ranks were mOTUs v.2.5.1, DUDes v.0.08, MetaPhIAn v.2.9.22, as well as the versions of the first CAMI challenge of DUDes v.0.08 and MetaPhIAn on marine data, together with DUDes v.cami1 and MetaPhIAn v.2.9.22 on strain madness data. On the plant-associated data, mOTUs v.cami1 and Bracken v.2.6 performed best with this metric (0.08 and 0.09).

\section{Difficult and easy taxa}

For all methods, viruses, plasmids, and Archaea were particularly difficult to detect (Supplementary Fig. 10, Supplementary Table 38) in the marine data. While many Archaeal taxa were detected by several methods, some taxa, such as Candidatus Nanohaloarchaeota, were not detected by any method in any sample. Similarly, no method detected any plasmids or viruses. In contrast, bacterial taxa in the Terrabacteria group and the phyla of Bacteroidetes and Proteobacteria were correctly detected by each method in all samples.

\section{Method similarity}

To assess software performances in relation to their methodological similarity, we clustered submissions based on the Bray Curtis dissimilarity on the vectors of precision and recall per taxa, averaged over ranks. Methods using similar information types, e.g., k-mer based (NBC++, Bracken), alignment (CCMetagen, Metalign), and marker gene approaches (mOTUs, MetaPhIAn) tended to cluster (Supplementary Fig. 11); for example, the two alignment-based approaches are more similar to each other than to other methods. Interestingly, the marker gene approaches are most similar to the gold standard, suggesting this class of methods is particularly well suited to infer taxonomic profiles. 


\section{Clinical pathogen prediction challenge}

A short-read metagenomic sequencing dataset of a blood sample from a patient with hemorrhagic fever of unknown cause was provided for participants to identify a causal pathogen together with further pathogens. Ten manually curated, hence not fully reproducible results were received (Supplementary Table 39). The total number of identified taxa per result varied considerably (Supplementary Fig. 12). Three submissions correctly identified the causal pathogen, Crimean-Congo hemorrhagic fever orthonairovirus (NCBI taxid 1980519), using the taxonomic profilers MetaPhIAn v.2.2, Bracken v.2.5, and CCMetagen v.1.1.367. Another submission using Bracken v.2.2 correctly identified orthonairovirus, but without indicating it as the causal pathogen.

\section{Computational requirements}

We measured the runtimes and maximum main memory usage for submitted methods across the marine and strain madness data (Fig. 5, Supplementary Table 40, Methods). Compute and memory efficient methods capable of processing the entire datasets within minutes to a few hours were available in every method category, even including some of the identified top ranked techniques. Substantial differences were seen within categories and even between versions, ranging from methods executable on standard desktop machines to those requiring extensive hardware and heavy parallelization. Of the assemblers, MEGAHIT was the fastest and most memory efficient, requiring $7 \mathrm{~h}$ and 42 GB of main memory to process marine short reads. This was $30 \%$ and $\sim 25 \%$ less time and memory than required by the second fastest and most memory efficient methods, OPERA-MS and GATB, respectively. On the marine assemblies, genome binners on average required $\sim 3 \mathrm{x}$ less time than for the smaller strain madness assemblies $(29.2 \mathrm{~h}$ vs. 86.1 h), but used almost $4 x$ more memory (69.9 GB vs. $18.5 \mathrm{~GB}$ ). MetaBAT 2.13.33 was the fastest (1.07 and $0.05 \mathrm{~h}$ ) and most memory efficient genome binner (max. memory usage $2.66 \mathrm{~GB}, 1.5 \mathrm{~GB}$ ) on both datasets. It was $\sim 5 \mathrm{x}$ and $\sim 635 \mathrm{x}$ faster than the second fastest method, Vamb fa045c0, $\sim 6 \mathrm{x}$ faster than LSHVec $1 \mathrm{dfe} 822$ on marine, and $765 x$ faster than SolidBin 1.3 on strain madness data; $\sim 2 x$ and $\sim 5 x$ more memory efficient than next ranking MaxBin 2.0.2 and CONCOCT 1.1.0 on marine data, 


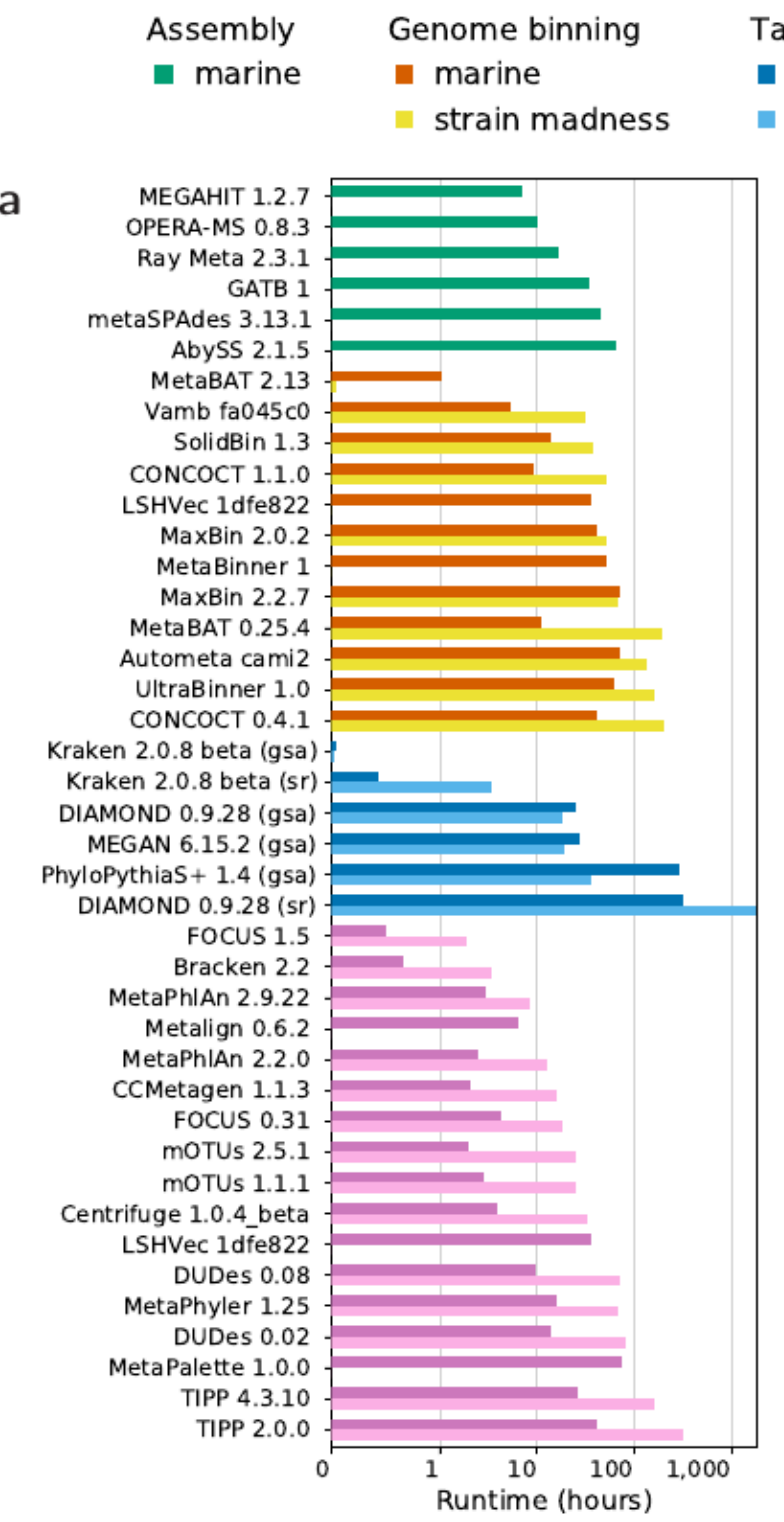
Taxonomic binning
marine
Taxonomic profiling
strain madness
- marine
- strain madness

a

b

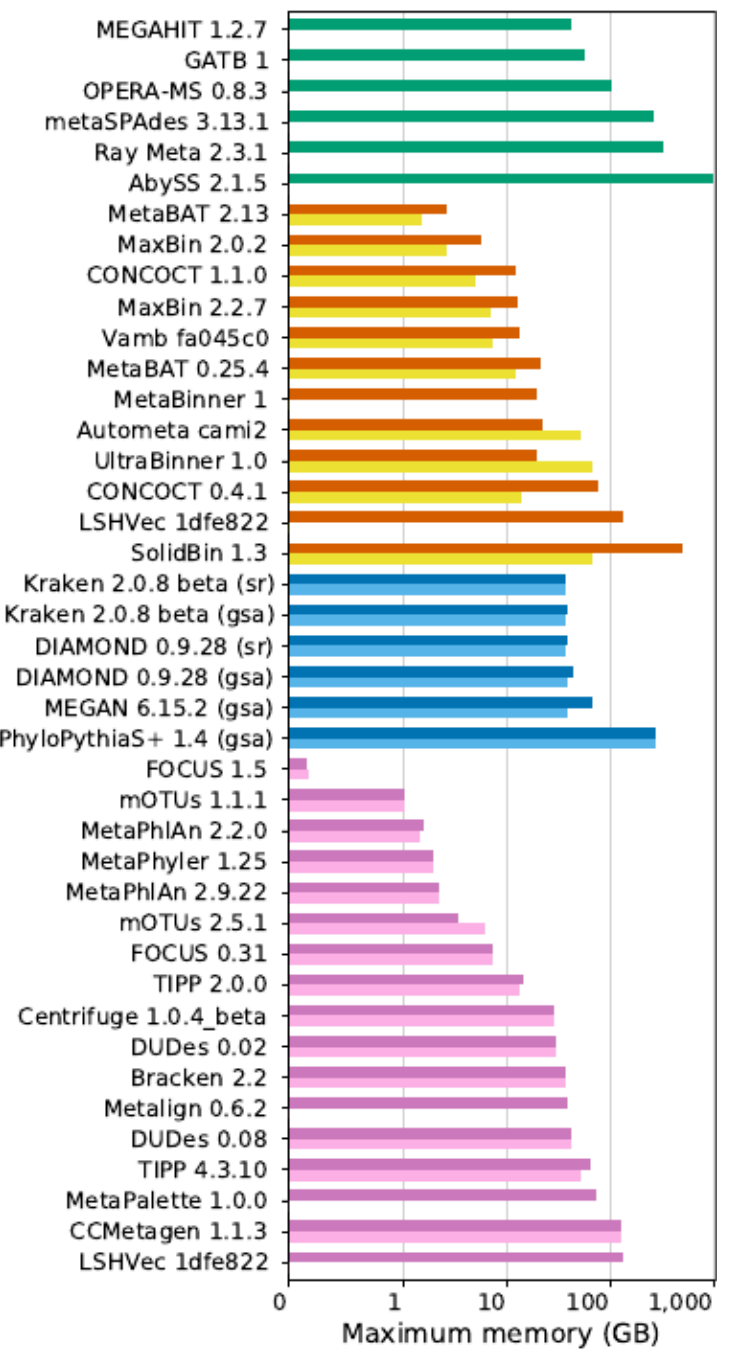

Fig. 5: Runtime (a) and maximum memory usage (b) of software from all categories for the marine and strain madness data (Supplementary Table 40) with log-scaled x-axis.

respectively. Both MetaBAT and CONCOCT were substantially ( 11x and $\sim 4 \mathrm{x})$ faster than the versions assessed in CAMI 1. Like genome binners, taxonomic binners ran longer on the marine than the strain madness assemblies, e.g., PhyloPythiaS+ with 287.3 vs. $36 \mathrm{~h}$, respectively, but had a similar or slightly higher memory usage. On the marine raw read data, taxon profilers, on the other hand, were almost $4 x$ faster on average (16.1 $\mathrm{h}$ vs. $60.8 \mathrm{~h}$ ) than on the $10 \mathrm{x}$ larger strain madness read dataset, but used more memory (38.1 GB vs. $25 \mathrm{~GB}$ ). The fastest and most memory efficient taxonomic binner was 
Kraken, requiring only 0.05 and $0.02 \mathrm{~h}$, respectively, and $\sim 37 \mathrm{~GB}$ memory on both datasets, for reads or contigs. It was followed by DIAMOND, which ran 500x and 910x as long on the marine and strain madness gold standard assemblies, respectively. FOCUS 1.5 and Bracken 2.2 were the fastest profilers on the marine $(0.51$ and $0.66 \mathrm{~h}$, respectively) and strain madness $(1.89,3.45 \mathrm{~h}$ ) data. FOCUS 1.5 also required the least memory (0.16 GB for marine, 0.17 GB for strain madness), followed by mOTUs 1.1 .1 and MetaPhIAn 2.2.0.

\section{Discussion and conclusions}

Assessing metagenomic analysis software thoroughly, comprehensively, and with little bias is key for optimizing data processing strategies as well as tackling open challenges in the field. In its second round, CAMI offered a diverse set of benchmarking challenges across a comprehensive data collection reflecting recent technical developments of the field. Here, we analyzed 5,002 results of 76 program versions with different parameter settings across 131 long and short read metagenome samples from four environments (marine, plant-associated, strain madness, clinical pathogen challenge). This effort increased the number of results $22 x$ and the number of benchmarked software versions $3 x$ in comparison to the first CAMI challenge, delivering extensive new insights into software performances and their interpretation across a wide range of conditions. By systematically assessing runtime and memory requirements, we added two more key performance dimensions to the benchmark, which are very important to consider, given the ever-increasing dataset sizes.

In comparison to software assessed in the first CAMI challenges, assembler performances rose for best performing methods by up to $20 \%$. Still, in the presence of closely related strains, assembly contiguity, the fractions of genomes recovered, and strain recall decreased, suggesting that most assemblers, sometimes intentionally ${ }^{31,34}$, did not accurately resolve strain variation, resulting in more fragmented, less strainspecific assemblies. In addition, genome coverage, parameter settings, and data preprocessing notably impacted assembly quality, while performances did not differ much across software versions. Most submitted metagenome assemblies used only short 
reads, and long and hybrid assemblies had no higher overall quality. Hybrid assemblies, however, were better for difficult to assemble regions, such as the 16S rRNA gene, recovering substantially more complete genes than most short-read submissions. Hybrid assemblers were also less affected by the presence of closely related strains in pooled samples, suggesting that the addition of long reads helps to distinguish individual strains.

In comparison to the first CAMI challenges, ensemble binners presented a development accompanied by substantial improvements across metrics in comparison to most individual methods. Overall, genome binners demonstrated variable performances across different metrics and dataset types, with both high strain diversity and assembly quality presenting challenges that substantially reduced performances relative to unique genome binning, even in the case of large sample numbers, such as for the strain madness dataset. Interestingly, for the plant-associated data, which also included plant host and 55 fungal genomes, given sufficient coverage, high-quality bins were obtained also for these taxa.

For taxonomic binners and profilers, highly performant and computationally efficient software was available, performing well across a range of conditions and metrics. Particularly the profiling field has matured in comparison to the first challenges, with less variance in top performing methods across taxon identification, abundance, and diversity estimates. Performance for both categories was found to be high for genus rank and above, with a substantial drop for bacterial species. As the second challenge data include high quality public genomes, the data is less divergent from publicly available data than for the first challenges, on which method performances declined already going from family to genus rank. It was also low for Archaea, viruses and plasmids, suggesting a need for developers to extend their focus in terms of reference sequence collections and model development. Another encouraging result is that the causal pathogen was successfully identified by several submissions in a clinical pathogen challenge. However, due to manual curation, none was reproducible, which indicates another area requiring improvement.

In its second challenge, CAMI identified key advances across common metagenomics software categories as well as current challenges for the field. As the state-of-the-art in 
methods and data generating techniques progresses, it will be important to continuously reevaluate these questions. In addition, computational methods for other microbiome data modalities $^{12}$ and multi-omics data integration could be jointly assessed. Most importantly, CAMI is a community-driven effort, and we encourage everyone with an interest in benchmarking from the field of microbiome research to join us.

\section{Methods}

\section{Community involvement}

We gathered community input on the nature and principles of implementing benchmarking challenges and datasets in public workshops and hackathons (https://www.microbiome-cosi.org/cami/participate/schedule). The most relevant metrics for performance evaluation and data interpretation were discussed in a public evaluation workshop with challenge participants and developers of evaluation software, where first challenge results were presented in an anonymized manner. Computational support for challenge participants was provided by the de.NBI cloud.

\section{Standardization and reproducibility}

To ensure reproducibility and assess computational behavior (runtimes and memory consumption) of the software used to create challenge submissions, we reproduced and reassessed the results according to submission specifications (Supplementary Table 2, https://data.cami-challenge.org/). For metagenome assemblers, computational requirements were assessed on a machine with Intel Xeon Processor $(2.6 \mathrm{GHz})$ virtualized to 56 cores (50 cores were used) and 2755 GB of main memory and for binners and profilers on a machine with an Intel Xeon E5-4650 v4 CPU (virtualized to 16 CPU cores, 1 thread per core) and 512 GB of main memory. We also updated Docker BioContainers implementing a range of commonly used performance metrics to include all metrics used in this evaluation (Supplementary Table 2). 


\section{Genome sequencing and assembly}

Illumina paired-end read data of 796 newly sequenced genomes, of which 224 stem from a Arabidopsis thaliana root environment, 176 from a marine environment ${ }^{73}, 384$ clinical $^{2}$ Streptococcus pneumoniae strains, and twelve strains from a murine gut environment were assembled using a pipeline with the SPAdes ${ }^{33}$ metagenome assembler (version 3.12). We removed contigs smaller than $1 \mathrm{~kb}$, as well as genome assemblies with a contamination of $5 \%$ or more and completeness of $90 \%$ or less, as determined with CheckM $^{74}$ version 1.011. Newly assembled and database genomes were taxonomically classified with CAMITAX ${ }^{75}$ and used as input for microbial community and metagenome data simulation with $\mathrm{CAMISIM}^{76}$, based on the from_profile mode for the marine and plant-associated dataset and the de novo mode for the strain madness datasets. All scripts and parameters for these steps are provided in the Supplementary Material and on

GitHub

(https://github.com/CAMI-

challenge/second challenge evaluation/tree/master/scripts/data generation).

For the plasmid dataset, inlet wastewater from a wastewater treatment plant on Zealand, Denmark was used to generate a plasmid sample similar to the procedure $\mathrm{in}^{77}$. Sequencing was performed on a NextSeq 500 on Nextera sequencing libraries (Illumina, San Diego, California, USA). A bioinformatic workflow described in ${ }^{78}$ was used to identify complete circular plasmids above $1 \mathrm{~kb}$ in size in the dataset.

\section{Challenge datasets}

For the challenges, participants were provided with long and short-read sequences for two metagenome datasets representing a marine and a plant associated environment, respectively, in complexity and taxonomic distribution, and for a "strain madness" dataset with very high strain diversity. Furthermore, a short-read clinical metagenomic dataset from a critically ill patient was provided.

The 10-sample $100 \mathrm{~Gb}$ marine dataset was created with CAMISIM from BIOM profiles of a deep-sea environment, using 155 newly sequenced marine isolate genomes from this environment and 622 genomes with matching taxonomic provenance from MarRef ${ }^{79}$, a 
manually curated database with completely sequenced marine genomes. Of these genomes, 303 (39.0\%, 204 of database genomes (31.9\%) and 99 new genomes (72.3\%)) had a closely related strain present, with an ANI of $95 \%$ or more. Additionally, 200 newly sequenced circular elements including plasmids and viruses were added. For each sample, $5 \mathrm{~Gb}$ of paired-end short Illumina and long Nanopore reads were created (Supplementary Text).

The 100-sample $400 \mathrm{~Gb}$ strain madness dataset includes 408 newly sequenced genomes, of which $97 \%$ (395) had a closely related strain. For each sample, $2 \mathrm{~Gb}$ of paired-end short and long-read sequences were generated with CAMISIM, respectively, using the same parameters and error profiles as in CAMI $1^{6}$ (Supplementary Text).

The 21-sample 315 Gb plant-associated dataset includes 894 genomes. Of these, 224 are from the proGenomes ${ }^{80}$ terrestrial representative genomes, 216 are newly sequenced genomes from an Arabidopsis thaliana root rhizosphere, 55 are fungal genomes associated with the rhizosphere ${ }^{81}, 398$ are plasmids or circular elements and one Arabidopsis thaliana genome. 15.3\% (137) of these genomes have at least one closely related genome present. For each sample, $5 \mathrm{~Gb}$ of paired-end short-read sequences, as well as 2x5 Gb long-read sequences mimicking Pacific Biosciences and Oxford Nanopore sequencing data, respectively, were generated. $90 \%$ of metagenome sequence data originate from bacterial genomes, $9 \%$ are fungal genome sequences, and $1 \%$ is from $A$. thaliana. To evaluate the assembly quality of single-sample versus cross-assembly strategies, 23 new genomes from eight clusters of closely related genomes were selected and added to the dataset in certain samples with predetermined abundances. For all three datasets, we generated gold standards for every metagenome sample individually and for the pooled samples, which included assemblies for short, long, and hybrid reads, genome bin and taxon bin assignments, and taxonomic profiles.

Finally, a $688 \mathrm{Mb}$ paired-end Miseq metagenomic sequencing dataset of a blood sample from a patient with hemorrhagic fever was provided. Previous analysis of the sample had revealed sequences matching the genome of Crimean-Congo hemorrhagic fever orthonairovirus (CCHFV), and the presence of the viral genome was subsequently 
confirmed via PCR (with a Ct value of 27.4). To create a realistic dataset and case for the challenge while protecting the identity of the patient, a clinical case description derived from the true anamnesis and modified in ways consistent with the causative agent was created. Additionally, all reads mapping to the human genome were replaced by sequences from the same genomic regions randomly drawn from the 1000 genomes dataset $^{82}$. Challenge participants were asked to identify the causal pathogen as well as all other pathogens present in the sample.

\section{Challenge organization}

The second round of CAMI challenges assessed software for metagenome assembly, genome binning, taxonomic binning, and taxonomic profiling. In addition, a diagnostic pathogen prediction challenge was provided. As before, two metagenome "practice" benchmark datasets were created from public genomes and provided together with the standard of truth before the challenges, to enable contest participants to familiarize themselves with data types and formats. These included a 49-sample dataset modelled from Human Microbiome data ${ }^{43}$ and a 64-sample dataset modelled in taxonomic composition from mouse gut samples ${ }^{83,84}$, with 5 Gb long (Pacific Biosciences, variable length with a mean of 3000 bp) and 5 Gb short (Illumina HiSeq2000, 150 bp) paired-end read sequences, respectively. Read profiles (read length and error rates) were created from sequencing runs on the MBARC-26 dataset ${ }^{85}$. Reference data collections with NCBI RefSeq, nr/nt and taxonomy from January 8th of 2019 were provided to participants, for use with reference-based methods in the challenges. For future benchmarking, use of these resources will facilitate method performance comparisons, as all genomes incorporated into the CAMI challenge datasets will be submitted to public sequence repositories.

The second CAMI challenge started on January 16th of 2019 (https://www.microbiomecosi.org/cami/cami/cami2). Participants registered for download of the challenge datasets, with 332 teams registering from that time until January 2021. For reproducibility, participants could submit either a Docker container containing the complete workflow, a bioconda script or a software repository with detailed installation instructions, specifying 
all parameter settings and reference databases used. Assembly results could be submitted for short-read data, long-read data, or both data types combined. For methods incapable of submitting a cross-sample assembly for the entire dataset, a cross-sample assembly for the first ten samples of a dataset could be submitted. Participants could also submit single-sample assemblies for each of the first five samples of a dataset. Specification of the performance criteria for strain-aware assembly can be found in the Supplementary Material. The assembly challenge closed on May 17, 2019. Immediately afterwards, gold standard and MEGAHIT ${ }^{31}$ assemblies were provided for both datasets. The gold standard assemblies include all sequences of the reference genomes and circular elements covered by one short read in the combined metagenome datasets. Analysis of gold standard assembly binnings allowed us to assess binning performances independently of assembly quality. We assessed the contributions of assembly quality by comparing with the binning results on MEGAHIT assemblies. Profiling results were submitted for all individual samples and for the entire datasets, respectively. Binning results included genome or taxon bin assignments for analyzed reads or contigs of the provided assemblies for every sample of a dataset. Results for the pathogen detection challenge included predictions of all pathogens and a causal pathogen responsible for the symptoms outlined in a clinical case description provided together with the clinical metagenome dataset. The CAMI II challenges ended on October 25, 2019. Subsequently, another round of challenges ("CAMI II b") on plant-associated data was offered starting on February 14, 2020. This closed on September 29, 2020 for assembly submissions and on January 31, 2021 for genome and taxonomic binning, as well as profiling.

Altogether 5,002 submissions of 76 programs were received for the four challenge datasets, from 30 external teams and CAMI developers (Supplementary Table 2). All genome data used for generation of the benchmark datasets as well as their metadata was kept confidential during the challenge and released afterwards (10.4126/FRL01006421672). To support an unbiased assessment, program submissions were represented with anonymous names in the portal (known only to submitters), and a second set of anonymous names for evaluation and discussion in the evaluation workshop, such that identities were unknown to all except for data analysis team (F.M., 
Z-L.D., A.F., A.S.), and program identities revealed only after a first consensus was reached.

\section{Evaluation metrics}

In the following, we briefly outline the metrics used to evaluate the four software categories. For details, the reader is also referred to $20,53,61$.

Assemblies: Assemblies were evaluated with metaQUAST 5.1.0rc using the --uniquemapping flag. This flag allows every contig to be mapped at only a single reference genome position. In evaluation, we focused on commonly used assembly metrics such genome fraction, mismatches per $100 \mathrm{~kb}$, duplication ratio, NGA50 and the number of misassemblies. The genome fraction specifies the percentage of reference bases covered by assembled contigs obtained by similarity-based mapping. Mismatches per $100 \mathrm{~kb}$ specify the number of mismatched bases in the contig-reference alignment. The duplication ratio is defined as the total number of aligned bases divided by genome fraction multiplied with reference length. NGA50 is a metric for measuring the contiguity of an assembly. For each reference genome, all contigs aligned to it are sorted by size and the NGA50 for that genome is defined as the length of the contig cumulatively surpassing $50 \%$ genome fraction. If a genome is not covered to $50 \%$, NGA50 is undefined. Since we report the average NGA50 over all genomes, it was set to 0 for genomes with less than $50 \%$ genome fraction. Finally, the number of misassemblies describes the number of contigs which either contain a gap of more than $1 \mathrm{~kb}$, contain inserts of more than $1 \mathrm{~kb}$ or align to different genomes. In addition to these metrics, we determined the strain recall and strain precision, similar to ${ }^{38}$, to quantify the presence of high-quality, strain-resolved assemblies. Strain recall is defined as the fraction of highquality (more than 90\% genome fraction and less than 100 mismatches per $100 \mathrm{~kb}$ ) genome assemblies recovered for all ground truth genomes. Strain precision specifies the fraction of high-quality assemblies among all high genome fraction (more than 90\%) assemblies.

Genome binning: for every predicted genome bin $b$, the true positives $T P_{b}$ are the number of base pairs of the most abundant genome $g$ in $b$, the false positives $F P_{b}$ are the number 
of base pairs in $b$ belonging to genomes other than $g$, and the false negatives $F N_{b}$ are the number of base pairs belonging to $g$ that are not in $b$.

Purity is defined for each predicted genome bin $b$ as:

purity $_{b}=\frac{T P_{b}}{T P_{b}+F P_{b}}$

The average purity is a simple average of the purity of bins $b$ in the set of all predicted genome bins $B$, that is:

average purity $=\frac{\Sigma_{b \in B} \text { purity }_{b}}{|B|}$.

Completeness is defined for each genome $g$ based on its mapping to a genome bin $b$ that it is most abundant in, as:

completeness $_{g b}=\frac{T P_{g b}}{T P_{g b}+F N_{g b}}$.

The average completeness is defined over all genomes in the sample, including those that are the most abundant in none of the predicted genome bins. Let $X$ be the set of such genomes. The average completeness is then defined as:

average completeness $=\frac{\Sigma_{b \in B} \text { completeness }_{g b}}{|B|+|X|}$.

As another metric, we consider the number of predicted genome bins that fulfill specific quality criteria. Bins with $>50 \%$ completeness and $<10 \%$ contamination are denoted as "moderate or higher" quality bins and bins with completeness $>90 \%$ and contamination $<5 \%$ as high-quality genome bins, similarly as in CheckM $^{74}$.

The Adjusted Rand index (ARI) is defined as in ${ }^{53}$. The Rand index compares two clusterings of the same set of items. Assuming the items are base pairs of different sequences, base pairs belonging to the same genome that were binned together in the same genome bin are considered true positives, and base pairs belonging to different genomes that were put into different genome bins are considered true negatives. The Rand index is the sum of true positives and negatives, divided by the total number of base 
pairs. The ARI is a normalized variant of the Rand index, such that the result ranges between 1 (best) and 0 (worst; see ${ }^{53}$ for a complete definition). As binning methods may leave a portion of the data unbinned, but the ARI is not suitable for datasets that are only partially assigned, it is computed for the binned portion only and interpreted together with the percentage of binned base pairs of a dataset.

Taxonomic binning: metrics are calculated for each of the major taxonomic ranks, from superkingdom or domain to species. Purity and completeness for each taxonomic bin $b$ (i.e., group of sequences and base pairs therein assigned to the same taxon) are computed by setting $T P_{b}$ to the number of base pairs of the true taxon $t$ assigned to $b$, $F P_{b}$ the number of base pairs assigned to $b$ belonging to other taxa, and $F N_{b}$ the number of base pairs of $t$ not assigned to $b$. The average purity at a certain taxonomic rank is a simple average of the purity of all predicted taxon bins at that taxonomic rank.

The average completeness at a certain taxonomic rank is the sum of the completeness over all predicted taxon bins divided by the number of taxa GS in the gold standard at that taxonomic rank. That is:

average completeness $=\frac{\Sigma_{b \in B} \text { completeness }_{b}}{|G S|}$.

The accuracy at a certain taxonomic rank is defined as:

accuracy $=\frac{\Sigma_{b \in B} T P_{b}}{n}$,

where $B$ is the set of predicted taxon bins at that taxonomic rank and $n$ is the total number of base pairs in GS for that taxonomic rank.

Average purity, completeness, and accuracy are also computed for a filtered subset $B_{f}$ of $B$ of each taxonomic rank, without the $1 \%$ smallest bins, and are denoted below ${\text { average } \text { purity }_{f} \text {, average completeness }}_{f}$, and accuracy $_{f} . B_{f}$ is obtained by sorting all bins in $B$ by increasing size in base pairs and filtering out the first bins whose cumulative size sum is smaller or equal to $1 \%$ of summed size of all bins in $B$. These metrics are then computed as: 
average purity pur $_{f}=\frac{\Sigma_{b \in B_{f}} \text { purity }_{b}}{\left|B_{f}\right|}$

average completeness ${ }_{f}=\frac{\Sigma_{b \in B_{f}} \text { completeness }_{b}}{|G S|}$,

$\operatorname{accuracy}_{f}=\frac{\Sigma_{b \in B_{f}} T P_{b}}{n}$

Taxonomic profiling: we determined purity and completeness in taxon identification, L1 norm and weighted UniFrac ${ }^{86}$ as abundance metrics, and alpha diversity estimates using the Shannon equitability index, as outlined below. We also calculated the following summary statistic: for each metric, we ranked the profilers by their average performance over samples. Each was assigned a score for its ranking ( 0 for first place among all tools at a particular taxonomic rank, 1 for second place, etc.). These scores were then added over the taxonomic ranks, from domain to species, to give an overall summary ranking score.

The purity and completeness for a taxonomic profile measure a method's ability to determine the presence and absence of taxa in a sample, at a certain taxonomic rank, without considering their relative abundances. Let the true positives $T P$ and false positives FP be the number of correctly and incorrectly detected taxa, that is, taxa present or absent in the gold standard profile, respectively, for a certain sample and rank. Further, let the false negatives $F N$ be the number of taxa that are in the gold standard profile but a method failed to detect. Purity, completeness, and F1-score are then defined as above.

The L1 norm error, Bray-Curtis distance, and weighted UniFrac error measure a method's ability to determine the relative abundances of taxa in a sample. Except for the UniFrac metric (which is rank independent), these are defined at each taxonomic rank. Let $x_{t}$ and $x_{t}^{*}$ be the true and predicted relative abundances of taxon $t$ in a sample, respectively. The L1 norm gives the total error between $x_{t}$ and $x_{t}^{*}$ in a sample, for all true and predicted $t$ at a certain rank and ranges between 0 and 2 . It is determined as:

\section{L1 norm error $=\Sigma_{t}\left|x_{t}-x_{t}^{*}\right|$}


The Bray-Curtis distance is the L1 norm error divided by the sum of all abundances $x_{t}$ and $x_{t}^{*}$ at the respective rank, that is:

Bray-Curtis distance $=\frac{\Sigma_{t}\left|x_{t}-x_{t}^{*}\right|}{\Sigma_{t} x_{t}+x_{t}^{*}}$

The Bray-Curtis distance ranges between 0 and 1. As the gold standards usually contain abundances for $100 \%$ of the data, it is equal to half of the L1 norm error if the profiler made predictions also for $100 \%$ of the data, and higher otherwise.

The weighted UniFrac metric uses differences between predicted and actual abundances weighted by distance in the taxonomic tree. It ranges between 0 (best) and 16 (worst). We use the EMDUnifrac implementation of the UniFrac distance ${ }^{87}$.

The Shannon equitability index is defined for each rank as:

Shannon equitability index $=\frac{\Sigma_{t} x_{t}^{*} \times \ln \left(x_{t}^{*}\right)}{\ln (m)}$,

where $m$ is the total number of taxa $t$. The index ranges from 0 to 1 , with 1 indicating complete evenness. As the diversity estimate is computed from a predicted profile alone, we assess its absolute difference to the index of the gold standard for comparison.

\section{Data availability and accession code availability statements}

A Life Science Reporting Summary for this paper will be made available. The benchmarking challenge and exemplary datasets (for developers to familiarize upfront with data types and formats) are available in PUBLISSO with the DOls 10.4126/FRL01006425521 (marine, strain madness, plant-associated), 10.4126/FRL01-006421672 (mouse gut), and 10.4126/FRL01-006425518 (human) and on the CAMI data portal for download (https://data.cami-challenge.org/participate). Datasets include gold standards, assembled genomes underlying benchmark data creation, NCBI taxonomy versions, and reference sequence collections for NCBI RefSeq, nt and nr (status 019/01/08). Benchmarked software outputs are available on Zenodo (https://zenodo.org/communities/cami/). Further software and scripts used for data analyses, and results are available at https://github.com/CAMI- 
challenge/second challenge evaluation. Supplementary Table 2 specifies the evaluated programs, parameters used, and installations options, including software repositories, Bioconda package recipes, Docker images, Bioboxes, and Biocontainers. Source data and scripts for Figures $1-5$ are available online (https://github.com/CAMlchallenge/second challenge evaluation/).

\section{Acknowledgements}

The authors thank all members of the metagenomics community who provided inputs and feedback on the project in public workshops and gratefully acknowledge funding of the DZIF (Project number TI 12.002_00; F.Mey.), German Excellence Cluster RESIST (EXC 2155 - Projektnummer 390874280; Z-.L.D.). D.K. was supported in part by the National Science Foundation under Grant No. 1664803; A.G. by St. Petersburg State University (grant ID PURE 73023672); D.A., A.Kor., D.M., and S.N. by the Russian Science Foundation (grant 19-14-00172); C.T.B. and L.I. in part by the Gordon and Betty Moore Foundation's Data-Driven Discovery Initiative through Grants GBMF4551 to C.T.B.; R.C. and R.V. by ANR Inception (ANR-16-CONV-0005) and PRAIRIE (ANR-19-P3IA-0001); S.D.K. by the European Research Council (ERC) under the European Union's Horizon 2020 research and innovation programme (ERC-COG-2018); J.K. and E.R.R. by the National Science Foundation under Grant No. 1845890; S.M. partially by National Science Foundation grants 2041984; V.R.M. by the Tony Basten Fellowship, Sydney Medical School Foundation. G.L.R. and Z.Z. partially by NSF grants 1936791 and 1919691; M.T. by the European Research Council (ERC) under the European Union's Horizon 2020 research and innovation programme (ERC-COG-2018); S.Z. by the Shanghai Municipal Science and Technology Commission [2018SHZDZX01], 111 Project [B18015]; S.Ha. by the Deutsche Forschungsgemeinschaft (DFG, German Research Foundation) through the '2125 DECRyPT' Priority Programme; R.E., E.Go., Zho.W., and A.T. by the DOE Office of Biological and Environmental Research under contract number DE-AC02-05CH11231. This research used resources of the National Energy Research Scientific Computing Center, which is supported by the Office of Science of the U.S. Department of Energy under Contract No. DE-AC02-05CH11231. The work conducted 
by the U.S. Department of Energy Joint Genome Institute, a DOE Office of Science User Facility, is supported under Contract No. DE-AC02-05CH11231.

\section{Author contributions}

F.Mey., A.F., Z.-L.D., D.K., M.A., D.A., F.B., D.B., J.J.B., C.T.B., J.B., A.Bu., B.C., R.C., P.T.L.C.C., A.C., R.E., E.E., E.Ge., E.Go., S.Ho., P.H., L.I., H.J., S.D.K., M.K., A.Kor., J.K., N.L., C.Le., C.Li., A.L., F.M.-M., S.M., V.R.M., C.M., P.M., D.M., D.R.M., A.M., N.N., J.N., S.N., L.O., L.P., P.P., V.C.P., J.S.P., S.Ras., E.R.R., K.R., B.R., G.L.R., H.-J.R., V.S., N.Se., E.S., L.S., F.S., S.S., A.T., C.T., M.T., J.T., G.U., Zho.W., Zi.W., Zhe.W., A.W., K.Y., R.Y., G.Z., Z.Z., S.Z., J.Z., and A.S. participated in challenge and created results; P.W.D., L.H.H., T.S.J., T.K., A.Kol., E.M.R., S.J.S., N.P.W., R.G.-O., P.G., S.Ha., S.Hä., A.Kh., F.Ma., F.Mes., S.Rad., P.S.-L., N.Sm., and T.S. generated and contributed data; A.F., A.Br., A.S., and A.C.M. generated benchmark datasets; F.Mey., D.K., A.G., M.A.G., L.I., G.L.R., Z.Z., and A.C.M. implemented benchmarking metrics; F.Mey., A.F., D.K., A.S., Z.-L.D., and A.C.M. performed evaluations and interpreted results with comments from many authors; F.Mey., A.F., Z.-L.D., D.K., A.G., G.R., F.B., R.C., P.W.D., A.E.D., R.E., D.R.M., A.M., E.R.R., B.R., G.L.R., H.-J.R., S.S., R.V., Z.Z., A.Br., A.S., and A.C.M made conceptual inputs to challenge design or evaluation; F.Mey., A.C.M., A.F., D.K. and Z.-L.D. wrote the paper with comments from many authors; A.S. and A.C.M. conceived the research with input from many authors.

\section{Competing interests}

A.E.D. co-founded Longas Technologies Pty Ltd, a company aimed at development of synthetic long-read sequencing technologies. 


\section{References}

1. Ghurye, J. S., Cepeda-Espinoza, V. \& Pop, M. Metagenomic Assembly: Overview, Challenges and Applications. Yale J. Biol. Med. 89, 353-362 (2016).

2. Breitwieser, F. P., Lu, J. \& Salzberg, S. L. A review of methods and databases for metagenomic classification and assembly. Brief. Bioinform. 20, 1125-1136 (2019).

3. Sangwan, N., Xia, F. \& Gilbert, J. A. Recovering complete and draft population genomes from metagenome datasets. Microbiome 4, 8 (2016).

4. Kumar Awasthi, M. et al. Metagenomics for taxonomy profiling: tools and approaches. Bioengineered 11, 356-374 (2020).

5. Maguire, F. et al. Metagenome-assembled genome binning methods with short reads disproportionately fail for plasmids and genomic Islands. Microb Genom 6, (2020).

6. Sczyrba, A. et al. Critical Assessment of Metagenome Interpretation-a benchmark of metagenomics software. Nat. Methods 14, 1063-1071 (2017).

7. Kastenmüller, G., Römisch-Margl, W., Wägele, B., Altmaier, E. \& Suhre, K. metaP-server: a web-based metabolomics data analysis tool. J. Biomed. Biotechnol. 2011, (2011).

8. Almeida, A., Mitchell, A. L., Tarkowska, A. \& Finn, R. D. Benchmarking taxonomic assignments based on $16 \mathrm{~S}$ rRNA gene profiling of the microbiota from commonly sampled environments. Gigascience 7, (2018).

9. Ye, S. H., Siddle, K. J., Park, D. J. \& Sabeti, P. C. Benchmarking Metagenomics Tools for Taxonomic Classification. Cell 178, 779-794 (2019).

10. Mclntyre, A. B. R. et al. Comprehensive benchmarking and ensemble approaches for metagenomic classifiers. Genome Biol. 18, 182 (2017).

11. Lindgreen, S., Adair, K. L. \& Gardner, P. P. An evaluation of the accuracy and speed of metagenome analysis tools. Sci. Rep. 6, 19233 (2016).

12. Van Den Bossche, T. et al. Critical Assessment of Metaproteome Investigation (CAMPI): A 
Multi-Lab Comparison of Established Workflows. bioRxiv 2021.03.05.433915 (2021) doi:10.1101/2021.03.05.433915.

13. Commichaux, S. et al. A critical assessment of gene catalogs for metagenomic analysis. Bioinformatics (2021) doi:10.1093/bioinformatics/btab216.

14. Wilkinson, M. D. et al. The FAIR Guiding Principles for scientific data management and stewardship. Sci Data 3, 160018 (2016).

15. Pasolli, E. et al. Extensive Unexplored Human Microbiome Diversity Revealed by Over 150,000 Genomes from Metagenomes Spanning Age, Geography, and Lifestyle. Cell 176, 649-662.e20 (2019).

16. Almeida, A. et al. A new genomic blueprint of the human gut microbiota. Nature 568, 499504 (2019).

17. Lesker, T. R. et al. An Integrated Metagenome Catalog Reveals New Insights into the Murine Gut Microbiome. Cell Rep. 30, 2909-2922.e6 (2020).

18. Bremges, A. \& McHardy, A. C. Critical Assessment of Metagenome Interpretation Enters the Second Round. mSystems 3, (2018).

19. Turnbaugh, P. J. et al. The human microbiome project. Nature 449, 804-810 (2007).

20. Meyer, F. et al. Tutorial: Assessing metagenomics software with the CAMI benchmarking toolkit. Cold Spring Harbor Laboratory 2020.08.11.245712 (2020) doi:10.1101/2020.08.11.245712.

21. Nawy, T. MICROBIOLOGY: The strain in metagenomics. Nat. Methods 12, 1005 (2015).

22. Segata, N. On the Road to Strain-Resolved Comparative Metagenomics. mSystems 3, (2018).

23. Anyansi, C., Straub, T. J., Manson, A. L., Earl, A. M. \& Abeel, T. Computational Methods for Strain-Level Microbial Detection in Colony and Metagenome Sequencing Data. Front. Microbiol. 11, 1925 (2020).

24. Simpson, J. T. et al. ABySS: a parallel assembler for short read sequence data. Genome 
Res. 19, 1117-1123 (2009).

25. Kolmogorov, M. et al. metaFlye: scalable long-read metagenome assembly using repeat graphs. Nat. Methods 17, 1103-1110 (2020).

26. Georganas, E. et al. HipMer: an extreme-scale de novo genome assembler. in Proceedings of the International Conference for High Performance Computing, Networking, Storage and Analysis 1-11 (Association for Computing Machinery, 2015).

27. Georganas, E. et al. Extreme Scale De Novo Metagenome Assembly. in SC18: International Conference for High Performance Computing, Networking, Storage and Analysis 122-134 (2018).

28. Hofmeyr, S. et al. Terabase-scale metagenome coassembly with MetaHipMer. Sci. Rep. 10, 10689 (2020).

29. Drezen, E. et al. GATB: Genome Assembly \& Analysis Tool Box. Bioinformatics 30, 29592961 (2014).

30. Chikhi, R. \& Rizk, G. Space-efficient and exact de Bruijn graph representation based on a Bloom filter. Algorithms Mol. Biol. 8, 22 (2013).

31. Li, D., Liu, C.-M., Luo, R., Sadakane, K. \& Lam, T.-W. MEGAHIT: an ultra-fast single-node solution for large and complex metagenomics assembly via succinct de Bruijn graph. Bioinformatics 31, 1674-1676 (2015).

32. Kieser, S., Brown, J., Zdobnov, E. M., Trajkovski, M. \& McCue, L. A. ATLAS: a Snakemake workflow for assembly, annotation, and genomic binning of metagenome sequence data. BMC Bioinformatics 21, 257 (2020).

33. Bankevich, A. et al. SPAdes: a new genome assembly algorithm and its applications to single-cell sequencing. J. Comput. Biol. 19, 455-477 (2012).

34. Nurk, S., Meleshko, D., Korobeynikov, A. \& Pevzner, P. A. metaSPAdes: a new versatile metagenomic assembler. Genome Res. 27, 824-834 (2017).

35. Bertrand, D. et al. Hybrid metagenomic assembly enables high-resolution analysis of 
resistance determinants and mobile elements in human microbiomes. Nat. Biotechnol. 37, 937-944 (2019).

36. Boisvert, S., Raymond, F., Godzaridis, E., Laviolette, F. \& Corbeil, J. Ray Meta: scalable de novo metagenome assembly and profiling. Genome Biol. 13, R122 (2012).

37. Mikheenko, A., Saveliev, V. \& Gurevich, A. MetaQUAST: evaluation of metagenome assemblies. Bioinformatics 32, 1088-1090 (2016).

38. Fritz, A. et al. Haploflow: Strain-resolved de novo assembly of viral genomes. bioRxiv (2021) doi:10.1101/2021.01.25.428049.

39. Bolger, A. M., Lohse, M. \& Usadel, B. Trimmomatic: a flexible trimmer for Illumina sequence data. Bioinformatics 30, 2114-2120 (2014).

40. Li, M., Copeland, A. \& Han, J. DUK - A Fast and Efficient Kmer Based Sequence Matching Tool. (2011).

41. Chen, S., Zhou, Y., Chen, Y. \& Gu, J. fastp: an ultra-fast all-in-one FASTQ preprocessor. Bioinformatics 34, i884-i890 (2018).

42. Nikolenko, S. I., Korobeynikov, A. I. \& Alekseyev, M. A. BayesHammer: Bayesian clustering for error correction in single-cell sequencing. BMC Genomics 14 Suppl 1, S7 (2013).

43. Human Microbiome Project Consortium. Structure, function and diversity of the healthy human microbiome. Nature 486, 207-214 (2012).

44. Kang, D. D., Froula, J., Egan, R. \& Wang, Z. MetaBAT, an efficient tool for accurately reconstructing single genomes from complex microbial communities. PeerJ 3, e1165 (2015).

45. Kang, D. D. et al. MetaBAT 2: an adaptive binning algorithm for robust and efficient genome reconstruction from metagenome assemblies. PeerJ 7, e7359 (2019).

46. Wu, Y.-W., Simmons, B. A. \& Singer, S. W. MaxBin 2.0: an automated binning algorithm to recover genomes from multiple metagenomic datasets. Bioinformatics 32, 605-607 (2016).

47. Alneberg, J. et al. Binning metagenomic contigs by coverage and composition. Nat. 
Methods 11, 1144-1146 (2014).

48. Miller, I. J. et al. Autometa: automated extraction of microbial genomes from individual shotgun metagenomes. Nucleic Acids Res. 47, e57 (2019).

49. Shi, L. \& Chen, B. A Vector Representation of DNA Sequences Using Locality Sensitive Hashing. bioRxiv 726729 (2019) doi:10.1101/726729.

50. Uritskiy, G. V., DiRuggiero, J. \& Taylor, J. MetaWRAP-a flexible pipeline for genomeresolved metagenomic data analysis. Microbiome 6, 158 (2018).

51. Wang, Z., Wang, Z., Lu, Y. Y., Sun, F. \& Zhu, S. SolidBin: improving metagenome binning with semi-supervised normalized cut. Bioinformatics 35, 4229-4238 (2019).

52. Nissen, J. N. et al. Improved metagenome binning and assembly using deep variational autoencoders. Nat. Biotechnol. (2021) doi:10.1038/s41587-020-00777-4.

53. Meyer, F. et al. AMBER: Assessment of Metagenome BinnERs. Gigascience 7, (2018).

54. Gregor, I., Dröge, J., Schirmer, M., Quince, C. \& McHardy, A. C. PhyloPythiaS+: a selftraining method for the rapid reconstruction of low-ranking taxonomic bins from metagenomes. PeerJ 4, e1603 (2016).

55. Wood, D. E., Lu, J. \& Langmead, B. Improved metagenomic analysis with Kraken 2. Genome Biol. 20, 257 (2019).

56. Buchfink, B., Reuter, K. \& Drost, H.-G. Sensitive protein alignments at tree-of-life scale using DIAMOND. Nat. Methods 18, 366-368 (2021).

57. Huson, D. H. et al. MEGAN Community Edition - Interactive Exploration and Analysis of Large-Scale Microbiome Sequencing Data. PLoS Comput. Biol. 12, e1004957 (2016).

58. Piro, V. C., Dadi, T. H., Seiler, E., Reinert, K. \& Renard, B. Y. ganon: precise metagenomics classification against large and up-to-date sets of reference sequences. Bioinformatics 36, i12-i20 (2020).

59. Zhao, Z., Cristian, A. \& Rosen, G. Keeping up with the genomes: efficient learning of our increasing knowledge of the tree of life. BMC Bioinformatics 21, 412 (2020). 
60. Sun, Z. et al. Challenges in benchmarking metagenomic profilers. Nat. Methods 18, 618$626(2021)$.

61. Meyer, F. et al. Assessing taxonomic metagenome profilers with OPAL. Genome Biol. 20, 51 (2019).

62. Milanese, A. et al. Microbial abundance, activity and population genomic profiling with mOTUs2. Nat. Commun. 10, 1014 (2019).

63. Kim, D., Song, L., Breitwieser, F. P. \& Salzberg, S. L. Centrifuge: rapid and sensitive classification of metagenomic sequences. Genome Res. 26, 1721-1729 (2016).

64. Segata, N. et al. Metagenomic microbial community profiling using unique clade-specific marker genes. Nat. Methods 9, 811-814 (2012).

65. Beghini, F. et al. Integrating taxonomic, functional, and strain-level profiling of diverse microbial communities with bioBakery 3. Elife 10, (2021).

66. Lu, J., Breitwieser, F. P., Thielen, P. \& Salzberg, S. L. Bracken: estimating species abundance in metagenomics data. (2017) doi:10.7717/peerj-cs.104.

67. Marcelino, V. R. et al. CCMetagen: comprehensive and accurate identification of eukaryotes and prokaryotes in metagenomic data. Genome Biol. 21, 103 (2020).

68. Piro, V. C., Lindner, M. S. \& Renard, B. Y. DUDes: a top-down taxonomic profiler for metagenomics. Bioinformatics 32, 2272-2280 (2016).

69. LaPierre, N., Alser, M., Eskin, E., Koslicki, D. \& Mangul, S. Metalign: efficient alignmentbased metagenomic profiling via containment min hash. Genome Biol. 21, 242 (2020).

70. Koslicki, D. \& Falush, D. MetaPalette: a k-mer Painting Approach for Metagenomic Taxonomic Profiling and Quantification of Novel Strain Variation. mSystems 1, (2016).

71. Liu, B., Gibbons, T., Ghodsi, M., Treangen, T. \& Pop, M. Accurate and fast estimation of taxonomic profiles from metagenomic shotgun sequences. BMC Genomics 12 Suppl 2, S4 (2011).

72. Pierce, N. T., Irber, L., Reiter, T., Brooks, P. \& Brown, C. T. Large-scale sequence 
comparisons with sourmash. F1000Res. 8, 1006 (2019).

73. Nguyen, T. T. \& Landfald, B. Polar front associated variation in prokaryotic community structure in Arctic shelf seafloor. Front. Microbiol. 6, 17 (2015).

74. Parks, D. H., Imelfort, M., Skennerton, C. T., Hugenholtz, P. \& Tyson, G. W. CheckM: assessing the quality of microbial genomes recovered from isolates, single cells, and metagenomes. Genome Res. 25, 1043-1055 (2015).

75. Bremges, A., Fritz, A. \& McHardy, A. C. CAMITAX: Taxon labels for microbial genomes. Gigascience 9, (2020).

76. Fritz, A. et al. CAMISIM: simulating metagenomes and microbial communities. Microbiome 7, 17 (2019).

77. Browne, P. D., Kot, W., Jørgensen, T. S. \& Hansen, L. H. The Mobilome: Metagenomic Analysis of Circular Plasmids, Viruses, and Other Extrachromosomal Elements. Methods Mol. Biol. 2075, 253-264 (2020).

78. Alanin, K. W. S. et al. An improved direct metamobilome approach increases the detection of larger-sized circular elements across kingdoms. Plasmid 115, 102576 (2021).

79. Klemetsen, T. et al. The MAR databases: development and implementation of databases specific for marine metagenomics. Nucleic Acids Res. 46, D692-D699 (2018).

80. Mende, D. R. et al. proGenomes2: an improved database for accurate and consistent habitat, taxonomic and functional annotations of prokaryotic genomes. Nucleic Acids Res. 48, D621-D625 (2020).

81. Durán, P. et al. Microbial Interkingdom Interactions in Roots Promote Arabidopsis Survival. Cell 175, 973-983.e14 (2018).

82. 1000 Genomes Project Consortium et al. A global reference for human genetic variation. Nature 526, 68-74 (2015).

83. Roy, U. et al. Distinct Microbial Communities Trigger Colitis Development upon Intestinal Barrier Damage via Innate or Adaptive Immune Cells. Cell Rep. 21, 994-1008 (2017). 
84. CAMI 2 - Multisample Benchmark Dataset of Mouse Gut.

https://repository.publisso.de/resource/frl:6421672.

85. Singer, E. et al. Next generation sequencing data of a defined microbial mock community. Sci Data 3, 160081 (2016).

86. Lozupone, C. \& Knight, R. UniFrac: a new phylogenetic method for comparing microbial communities. Appl. Environ. Microbiol. 71, 8228-8235 (2005).

87. McClelland, J. \& Koslicki, D. EMDUniFrac: exact linear time computation of the UniFrac metric and identification of differentially abundant organisms. J. Math. Biol. 77, 935-949 (2018). 